\title{
Higgs Parity grand unification
}

\author{
Lawrence J. Hall ${ }^{a, b}$ and Keisuke Harigaya ${ }^{c}$ \\ ${ }^{a}$ Department of Physics, University of California, \\ Berkeley, California 94720, U.S.A. \\ ${ }^{b}$ Theoretical Physics Group, Lawrence Berkeley National Laboratory, \\ Berkeley, California 94720, U.S.A. \\ ${ }^{c}$ School of Natural Sciences, Institute for Advanced Study, \\ Princeton, New Jersey 08540, U.S.A. \\ E-mail: ljhall@lbl.gov, keisukeharigaya@ias.edu
}

ABSTRACT: The vanishing of the Higgs quartic coupling of the Standard Model at high energies may be explained by spontaneous breaking of Higgs Parity. Taking Higgs Parity to originate from the Left-Right symmetry of the $\mathrm{SO}(10)$ gauge group, leads to a new scheme for precision gauge coupling unification that is consistent with proton decay. We compute the relevant running of couplings and threshold corrections to allow a precise correlation among Standard Model parameters. The scheme has a built-in solution for obtaining a realistic value for $m_{b} / m_{\tau}$, which further improves the precision from gauge coupling unification, allowing the QCD coupling constant to be predicted to the level of $1 \%$ or, alternatively, the top quark mass to $0.2 \%$. Future measurements of these parameters may significantly constrain the detailed structure of the theory. We also study an $\mathrm{SO}(10)$ embedding of quark and lepton masses, showing how large neutrino mixing is compatible with small quark mixing, and predict a normal neutrino mass hierarchy. The strong CP problem may be explained by combining Higgs Parity with space-time parity.

KEYwords: Beyond Standard Model, GUT, Quark Masses and SM Parameters

ARXiv EPRINT: 1905.12722 


\section{Contents}

1 Introduction 2

2 Higgs quartic coupling and Higgs Parity 4

3 Grand unification and the strong CP problem 5

3.1 Left-right symmetry as Higgs Parity 5

$\begin{array}{lll}3.2 & \text { Yukawa couplings and the strong CP problem } & 6\end{array}$

$\begin{array}{lll}3.3 & \mathrm{SO}(10) \text { unification } & 6\end{array}$

3.4 Degree of fine-tuning $\quad 7$

4 Gauge coupling unification and parity breaking scale $\quad 7$

$4.1 \mathrm{SU}(3) \times \mathrm{SU}(2) \times \mathrm{SU}(2) \times \mathrm{U}(1) \quad 8$

$\begin{array}{lll}4.2 & \mathrm{SU}(4) \times \mathrm{SU}(2) \times \mathrm{SU}(2) & 10\end{array}$

5 Yukawa couplings $\quad 12$

$\begin{array}{lll}5.1 & \text { Up-type quark yukawa couplings } & 12\end{array}$

$\begin{array}{lll}5.2 & \text { Down-type quark yukawa coupling } & 14\end{array}$

$\begin{array}{lll}5.3 & \text { Charged lepton yukawa couplings } & 14\end{array}$

$\begin{array}{ll}5.4 \text { Neutrino masses and mixing } & 15\end{array}$

$\begin{array}{ll}5.5 \text { A simple } \mathrm{SO}(10) \text { theory of flavor } & 16\end{array}$

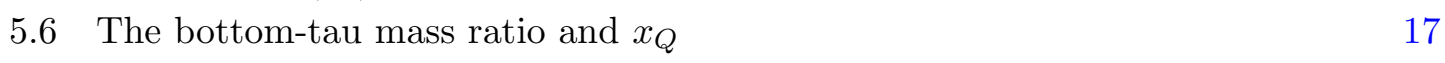

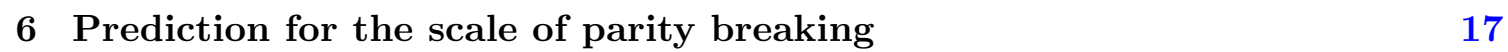

$\begin{array}{ll}\text { 6.1 Threshold corrections to the SM quartic coupling } & 17\end{array}$

$\begin{array}{lll}\text { 6.1.1 Threshold correction from charged gauge bosons } & 18\end{array}$

$\begin{array}{lll}\text { 6.1.2 Threshold correction from neutral gauge bosons } & 18\end{array}$

$\begin{array}{ll}\text { 6.1.3 Threshold correction from top quarks } & 19\end{array}$

6.1.4 Threshold correction from other fermions 20

6.1.5 Threshold correction from colored Higgses in the 422 theory 20

6.2 Top quark mass, QCD coupling and the Higgs Parity breaking scale 21

$\begin{array}{lll}7 & \text { Precise unification and SM parameters } & 21\end{array}$

$\begin{array}{lll}7.1 & \mathrm{SU}(3) \times \mathrm{SU}(2) \times \mathrm{SU}(2) \times \mathrm{U}(1) & 22\end{array}$

$7.2 \quad \mathrm{SU}(4) \times \mathrm{SU}(2) \times \mathrm{SU}(2) \quad 24$

8 Discussion $\quad 24$

A Contributions of $X$ states to beta functions 26

B Threshold corrections from SO(10) breaking scalars $\quad 27$

B.1 $\mathrm{SU}(3) \times \mathrm{SU}(2) \times \mathrm{SU}(2) \times \mathrm{U}(1) \quad 27$

B.2 $\mathrm{SU}(4) \times \mathrm{SU}(2) \times \mathrm{SU}(2) \quad 29$ 


\section{Introduction}

The discoveries of a perturbative Higgs boson at the Large Hadron Collider [1, 2] and no new states beyond the Standard Model (SM) [3, 4] suggest that the SM may be the correct effective theory of particle physics up to a scale orders of magnitude larger than the weak scale, a possibility largely ignored before the Large Hadron Collider. In such a scenario, progress in particle physics will depend on both precision measurements of SM parameters, as well as searches for rare processes, for example those violating baryon number, lepton numbers and CP.

Precision measurements can probe particle physics to extremely high energies. In 1974 Georgi, Quinn and Weinberg proposed that measurements of the three gauge couplings of the SM, $g_{1,2,3}$, could test whether the three gauge forces of nature are unified into a single grand unified gauge force with coupling strength, $g_{u}$, at some very high unified mass scale $M_{u}[5]$. The two fundamental UV parameters lead to a correlation among the three measured gauge couplings: $\left(\alpha_{u}, M_{u}\right) \rightarrow\left\{g_{1,2,3}\right\}$. After decades of measurements, this correlation is at best a first order approximation, requiring very large threshold corrections from the unified scale to force the low energy gauge couplings to meet and to make $M_{u}$ sufficiently large to be consistent with the experimental limit on the proton lifetime. Similarly, the simplest $\mathrm{SU}(5)$ [6] prediction for fermion masses, the ratio $m_{b} / m_{\tau}$ [7], is also at best a first order result, requiring large corrections. Nevertheless, unification is a bold and exciting vision that explains the gauge quantum numbers of the quarks and leptons, including charge quantization, and can be probed via precision measurements of SM parameters at low energy.

Precision measurements of the weak mixing angle at LEP [8] supported supersymmetric unification. Triggering the weak scale from supersymmetry breaking, $v \sim m_{\text {susy }}$, gave a successful correlation of the low energy gauge couplings via $\left(g_{u}, M_{u}, m_{\text {susy }} / v \simeq 1\right) \rightarrow$ $\left\{g_{1,2,3}\right\}$ [9-14]. While theories with a sufficiently long proton lifetime were easily constructed, the absence of superpartners at the Large Hadron Collider now makes it difficult to identify $m_{\text {susy }}$ with the weak scale, weakening the theoretical basis for this correlation.

With a $125 \mathrm{GeV}$ Higgs and the SM valid to sufficiently high energies, the Higgs quartic coupling of the SM passes through zero at a scale of order $\left(10^{9}-10^{12}\right) \mathrm{GeV}$ [15], as shown in figure 1. This very striking behavior suggests that new physics lies at the scale where the Higgs quartic coupling vanishes, and that this new physics should explain the vanishing quartic via a new symmetry. One possibility is that the new symmetry is supersymmetry; although the vanishing of the quartic is not guaranteed, it does occur in a large portion of parameter space $[16,17]$. We have recently introduced another possibility, "Higgs Parity" [18], that interchanges the weak SU(2) gauge group (and SM Higgs, $H$ ) with a partner gauge group $\mathrm{SU}(2)^{\prime}$ (and partner Higgs, $H^{\prime}$ )

$$
\mathrm{SU}(2) \leftrightarrow \mathrm{SU}(2)^{\prime} \quad H(2,1) \leftrightarrow H^{\prime}(1,2),
$$

where the quantum numbers of $H$ and $H^{\prime}$ refer to $\left(\mathrm{SU}(2), \mathrm{SU}(2)^{\prime}\right)$. Spontaneously breaking $\mathrm{SU}(2)^{\prime}$ by $\left\langle H^{\prime}\right\rangle=v^{\prime}$ leads to the Higgs being a Nambu-Goldstone boson with $\lambda_{\mathrm{SM}}\left(v^{\prime}\right)=0$ at 


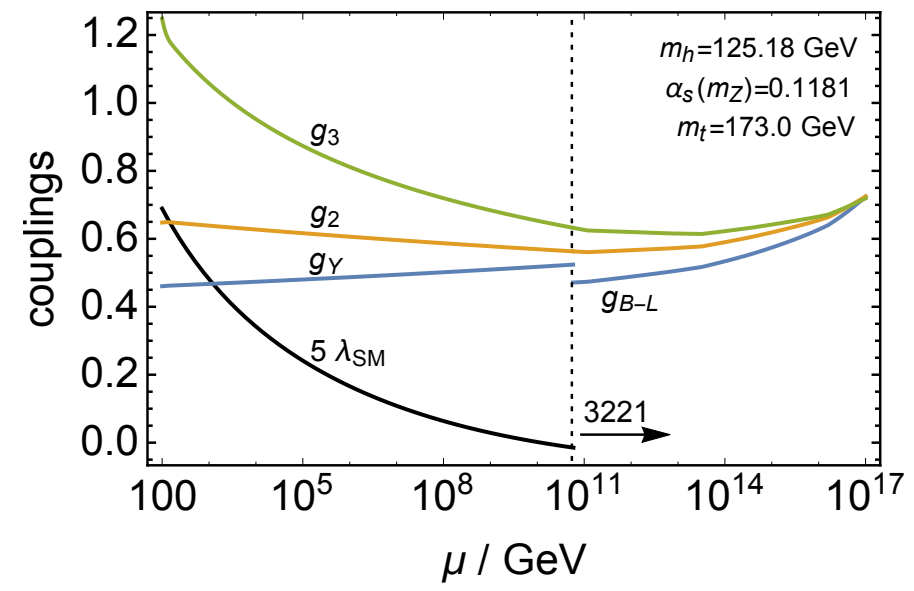

Figure 1. Precise gauge coupling unification via Higgs Parity. The intermediate scale is the energy scale where the running Higgs quartic coupling of the Standard Model nearly vanishes.

tree-level. Depending on the implementation, this can also solve the strong CP problem [18] and lead to interesting dark matter candidates [19].

In this paper we identify $\mathrm{SU}(2) \times \mathrm{SU}(2)^{\prime}$ as the $\mathrm{SU}(2)_{L} \times \mathrm{SU}(2)_{R}$ subgroup of the unified $\mathrm{SO}(10)$ gauge group [20,21], so that $v^{\prime}$ is identified as the scale of Left-Right symmetry breaking. In $\mathrm{SO}(10)$ unification, an intermediate scale of symmetry breaking introduces an extra free parameter so that the correlation of $\left\{g_{1,2,3}\right\}$ from gauge coupling unification is lost. However, in theories with Higgs Parity, $v^{\prime}$ is predicted from the Higgs mass so that a correlation is recovered, as illustrated in figure 1; three parameters of the unified theory yield a correlation among four measured observables, $\left(g_{u}, M_{u}, v^{\prime}\right) \rightarrow\left\{g_{1,2,3}, m_{h}\right\}$. In fact, the uncertainty in this correlation is dominated by the top quark Yukawa coupling $y_{t}$ via renormalization of the quartic coupling, so that in Higgs Parity Unification four UV parameters of the theory yield a correlation among five low energy observables [18]

$$
\left(g_{u}, M_{u}, y_{t}, v^{\prime}\right) \rightarrow\left\{g_{1,2,3}, m_{h}, m_{t}\right\} .
$$

Fixing three of the observables to their central measured values, allows a projection of this correlation into a two-dimensional subspace, as shown for $\left(m_{h}, \alpha_{s}\right)$ and $\left(m_{t}, \alpha_{s}\right)$ in the left and right panels of figure 2. The blue shaded region allows for threshold corrections at the unification scale with $\Delta<10$ (see eq. (4.10)). The black rectangles show the observed SM values. In figures 1 and 2 the gauge group above $v^{\prime}$ is $\mathrm{SU}(3) \times \mathrm{SU}(2)_{L} \times \mathrm{SU}(2)_{R} \times \mathrm{U}(1)_{B-L}$.

The organization of the paper is as follows. Sections 2 and 3 summarize the essence of Higgs Parity unification. In section 2, we review how Higgs Parity explains the vanishing of the SM Higgs quartic at a high scale. Section 3 discusses the embedding of Higgs Parity into $\mathrm{SO}(10)$ unified theories and how gauge coupling unification is tied to the vanishing quartic coupling. Sections 4-7 analyze the framework in more detail. Section 4 examines the running of gauge couplings between electroweak and unified scales, including threshold corrections at the unification scale, and derives the Higgs Parity symmetry breaking scale required for successful precision gauge coupling unification. The generation of the SM 

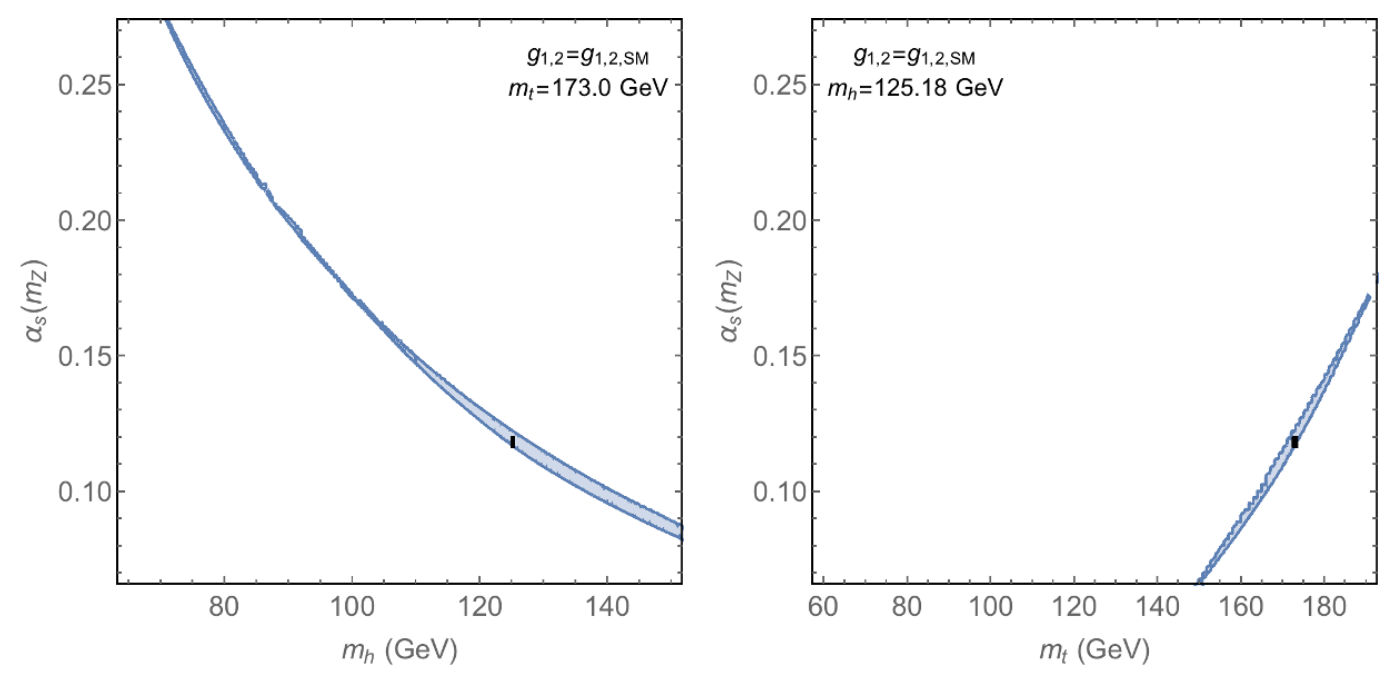

Figure 2. Correlation of low energy parameters from coupling unification with Higgs Parity, projected into the $\left(m_{h}, \alpha_{s}\right)$ and $\left(m_{t}, \alpha_{s}\right)$ planes.

fermion masses is discussed in section 5 . We show how the $b / \tau$ mass ratio and the structure of neutrino masses arise from an $\mathrm{SO}(10)$ unified theory. In section 6 , we derive the threshold corrections to the SM Higgs quartic coupling at the Higgs Parity symmetry breaking scale, and show the relation between $\left(m_{t}, \alpha_{s}\right)$ and the Higgs Parity symmetry breaking scale. Finally, the prediction for $\left(m_{t}, \alpha_{s}\right)$ from the precise coupling unification is given in section 7 .

\section{Higgs quartic coupling and Higgs Parity}

In this section we review the relation between the nearly vanishing SM Higgs quartic coupling at high energy scales and the Higgs Parity symmetry breaking scale introduced in [18]. Consider a $Z_{2}$ symmetry which exchanges the SM SU(2) gauge symmetry with a new gauge interaction $\mathrm{SU}(2)^{\prime}$, as well as the SM Higgs field $H(2,1)$ with its partner $H^{\prime}(1,2)$. Here the brackets show the $\mathrm{SU}(2) \times \mathrm{SU}(2)^{\prime}$ quantum numbers. We refer to this $Z_{2}$ symmetry as Higgs Parity.

Well below the cut off scale, the following renormalizable scalar potential dominates the dynamics of $H$ and $H^{\prime}$,

$$
V\left(H, H^{\prime}\right)=-m^{2}\left(\left|H^{2}\right|+\left|H^{\prime}\right|^{2}\right)+\lambda\left(|H|^{2}+\left|H^{\prime}\right|^{2}\right)^{2}+\lambda^{\prime}|H|^{2}\left|H^{\prime}\right|^{2} .
$$

We assume $m^{2}>0$ and $m \gg v$, the electroweak scale. Higgs Parity is spontaneously broken by the vacuum expectation value (VEV) $\left\langle H^{\prime}\right\rangle \equiv v^{\prime}$, with $v^{\prime 2}=m^{2} / 2 \lambda$. After integrating out $H^{\prime}$, the low energy effective potential of $H$ is

$$
V_{L E}(H)=\lambda^{\prime} v^{2}|H|^{2}-\lambda^{\prime}\left(1+\frac{\lambda^{\prime}}{4 \lambda}\right)|H|^{4} .
$$

To obtain the hierarchy $\langle H\rangle \ll v^{\prime}$, it is necessary to take a very small value of $\lambda^{\prime} \sim-v^{2} / v^{\prime 2}$, leading to a small value of the SM Higgs quartic coupling $\lambda_{\mathrm{SM}} \simeq 0$. This is the boundary 


\begin{tabular}{|c|c|c|c|c|c|c|}
\hline & $q$ & $\ell$ & $(\bar{u}, \bar{d})=\bar{q}$ & $(N, \bar{e})=\bar{\ell}$ & $H$ & $H^{\prime}$ \\
\hline $\mathrm{SU}(3)_{c}$ & 3 & 1 & $\overline{3}$ & 1 & 1 & 1 \\
\hline $\mathrm{SU}(2)_{L}$ & 2 & 2 & 1 & 1 & 2 & 1 \\
\hline $\mathrm{SU}(2)_{R}$ & 1 & 1 & 2 & 2 & 1 & 2 \\
\hline $\mathrm{U}(1)_{B-L}$ & $1 / 6$ & $-1 / 2$ & $-1 / 6$ & $1 / 2$ & $-1 / 2$ & $1 / 2$ \\
\hline 422 & \multicolumn{2}{|c|}{$(4,2,1)$} & \multicolumn{2}{|c|}{$(\overline{4}, 1,2)$} & $(4,2,1)$ & $(\overline{4}, 1,2)$ \\
\hline $\mathrm{SO}(10)$ & \multicolumn{4}{|c|}{16} & \multicolumn{2}{|c|}{16} \\
\hline
\end{tabular}

Table 1. The gauge charges of SM fermions, $H$ and $H^{\prime}$ under 3221 or 422.

condition on $\lambda_{\mathrm{SM}}$ at the renormalization scale $\mu_{c}=v^{\prime}$. Renormalization group running from the top quark yukawa makes $\lambda_{\mathrm{SM}} \simeq 0.1$ around the electroweak scale. From the IR perspective, the scale $v^{\prime}$ is identified with the energy scale around which the SM Higgs quartic coupling vanishes. Threshold corrections to $\lambda_{\mathrm{SM}}\left(v^{\prime}\right)$ as well as a precise prediction for $v^{\prime}$ are presented in section 6 .

In this paper, we identify Higgs Parity with the Left-Right symmetry which can be embedded into $\mathrm{SO}(10)$ grand unification. As we illustrated in the introduction and will elaborate in section 7 , this identification leads to a non-trivial scheme for precise gauge coupling unification.

\section{Grand unification and the strong CP problem}

\subsection{Left-right symmetry as Higgs Parity}

Let us first embed Higgs Parity into the Left-Right symmetry where $\mathrm{SU}(2)^{\prime}$ is identified with $\mathrm{SU}(2)_{R}$. The gauge symmetry above the scale $v^{\prime}$ is $\mathrm{SU}(3)_{c} \times \mathrm{SU}(2)_{L} \times \mathrm{SU}(2)_{R} \times \mathrm{U}(1)_{B-L}$ or $\mathrm{SU}(4) \times \mathrm{SU}(2)_{L} \times \mathrm{SU}(2)_{R}$, which we refer to as 3221 or 422 for short. 422 is the Pati-Salam gauge group [22], and $\mathrm{SU}(3)_{c} \times \mathrm{U}(1)_{B-L}$ is a subgroup of $\mathrm{SU}(4)$. The gauge quantum numbers of SM fermions, $H$ and $H^{\prime}$ are shown in table 1. The Left-Right symmetry, which we denote as $C_{L R}$, is

$$
\begin{gathered}
q \leftrightarrow \bar{q}, \quad \ell \leftrightarrow \bar{\ell}, \quad H \leftrightarrow H^{\prime}, \\
\mathrm{SU}(2)_{L} \leftrightarrow \mathrm{SU}(2)_{R}, \quad \text { charge conjugation on } \mathrm{SU}(3)_{c} \times \mathrm{U}(1)_{B-L} \text { or } \mathrm{SU}(4),
\end{gathered}
$$

and includes Higgs Parity. This results in the Higgs having gauge quantum numbers identical to leptons, which is not standard for Left-Right theories [23-28]. The 3221 or 422 gauge groups are broken down to the $\mathrm{SU}(3)_{c} \times \mathrm{SU}(2)_{L} \times \mathrm{U}(1)_{Y}$ group by the VEV of $H^{\prime}$.

We may also combine Left-Right symmetry with another discrete $Z_{2}$ symmetry; the most interesting option being space-time parity,

$$
\begin{aligned}
& q(t, x) \leftrightarrow i \sigma_{2} \bar{q}^{*}(t,-x), \quad \ell(t, x) \leftrightarrow i \sigma_{2} \bar{\ell}^{*}(t,-x), \quad H(t, x) \leftrightarrow H^{\prime *}(t,-x), \\
& \mathrm{SU}(2)_{L} \leftrightarrow \mathrm{SU}(2)_{R}, \quad \text { parity transformation on gauge fields, }
\end{aligned}
$$

which we denote as $P_{L R}$. As we will see, the strong CP problem may then be solved. 


\subsection{Yukawa couplings and the strong CP problem}

The gauge charges in table 1 forbid renormalizable yukawa couplings. Instead, the SM fermion masses arise from the mixing of $(f ; \bar{f})=(q, \ell ; \bar{q}, \bar{\ell})$ with extra massive fermions $(X ; \bar{X})$ via yukawa couplings and masses of the form

$$
\begin{gathered}
{\left[f_{i} x_{i j} \bar{X}_{j} H^{(\dagger)}+\bar{f}_{i} x_{i j}^{\prime} X_{j} H^{\prime(\dagger)}\right] \quad \text { or } \quad\left[f_{i} x_{i j} \bar{X}_{j} H^{\prime(\dagger)}+\bar{f}_{i} x_{i j}^{\prime} X_{j} H^{(\dagger)}\right]} \\
+m_{X, i j} X_{i} \bar{X}_{j} .
\end{gathered}
$$

Higgs Parity (and space-time parity) requires that $x_{i j}=x_{j i}^{(*)}$ and $m_{X, i j}=m_{X, j i}^{(*)}$. After $H^{\prime}$ obtains a VEV, $(f, \bar{f})$ mixes with $(X, \bar{X})$. A linear combination of them remains massless and has the yukawa coupling $f_{\mathrm{SM}_{i}} y_{i j} \bar{f}_{\mathrm{SM}_{j}} H^{(\dagger)}$. If the mass of $X$ is much larger than $x v^{\prime}$, we may integrate out $X$ to obtain a dimension-five operator $f \bar{f} H^{(\dagger)} H^{\prime(\dagger)}$, which yields a yukawa coupling $y \sim x^{2} v^{\prime} / m_{X}$. For the top yukawa this is not a good description as $m_{X} \sim x v^{\prime}$, and diagonalization of the mass matrix is required to extract the top yukawa, which is done in section 5 .

The strong CP problem can be solved by combining Left-Right symmetry with spacetime parity, as the symmetry forbids the $\theta$ term and constrains the determinant of the quark mass matrix [23, 24]. See refs. [29-38] for studies on Left-Right symmetric solutions to the strong CP problem. Refs. [39, 40] propose a model with a structure for yukawa couplings similar to ours and show that the strong CP problem is actually solved since $x_{i j}^{\prime}=x_{i j}^{*}$ and $m_{X, i j}$ is Hermitian. They obtain the hierarchy $v \ll v^{\prime}$ by softly breaking the Left-Right symmetry with space-time parity. In out setup the symmetry, which we call Higgs Parity, is spontaneously broken without soft breaking, predicting a vanishing $\lambda_{\mathrm{SM}}\left(v^{\prime}\right)$. Spontaneous breaking of Higgs Parity generates a phase in the determinant of the quark mass matrix via two-loop quantum corrections [18]. Assuming that the couplings $x$ are $O(1)$, the corrections are safely below the current limit from the neutron electric dipole moment, but in the range that can be probed by planned experiments. The model of flavor presented in section 5 does not obey this assumption, and the corrections may be larger.

\section{3 $\mathrm{SO}(10)$ unification}

The 3221 and 422 theories can both be embedded into $\mathrm{SO}(10)$ grand unified theories. The $\mathrm{SO}(10)$ gauge charges of the SM fermions, $H$ and $H^{\prime}$ are shown in table 1 . The SM fermions are unified into three $\mathbf{1 6} \mathrm{s}$, and the Higgs fields $H$ and $H^{\prime}$ are also embedded into a $\mathbf{1 6}$.

The symmetry breaking pattern is

$$
\mathrm{SO}(10) \rightarrow\left\{\begin{array}{l}
\mathrm{SU}(3) \times \mathrm{SU}(2)_{L} \times \mathrm{SU}(2)_{R} \times \mathrm{U}(1)_{B-L} \quad \stackrel{H^{\prime}}{\rightarrow} \quad \mathrm{SU}(3) \times \mathrm{SU}(2)_{L} \times \mathrm{U}(1)_{Y} . \\
\mathrm{SU}(4) \times \mathrm{SU}(2)_{L} \times \mathrm{SU}(2)_{R}
\end{array} \quad\right.
$$

The theory has three UV parameters relevant for gauge coupling unification: the $\mathrm{SO}(10)$ gauge coupling, the $\mathrm{SO}(10)$ symmetry breaking scale, and the LR symmetry breaking scale $v^{\prime}$. As there are also three SM gauge coupling constants, it is not surprising that one can typically find a set of the three UV parameters that allow coupling unification. However, as we have shown, the LR symmetry breaking scale is not a free parameter when it is linked to 
Higgs Parity breaking, but is determined by the running of the SM Higgs quartic coupling. In this case, it would be significant if coupling unification were successful. In figure 1, we fix the scale $v^{\prime}$ using the central values of the Higgs mass, top quark mass and QCD coupling shown in the figure, and solve the RGE equations assuming the 3221 theory. Remarkably, gauge coupling unification occurs, and at a scale consistent with the proton lifetime.

In section 7 , we analyze the precision of this coupling unification, including threshold corrections to gauge coupling constants at the unification scale, as well as the threshold corrections to the SM quartic coupling at the scale $v^{\prime}$. The unification of the yukawa couplings is discussed in section 5 .

\subsection{Degree of fine-tuning}

We comment on the fine-tuning of parameters in the Higgs potential (2.1) required for symmetry breaking. First, $m^{2}$ must be fine-tuned by an amount $\Delta_{m^{2}}$, so that the Higgs Parity breaking scale $v^{\prime}$ is much less than the cutoff scale $\Lambda$, which must be larger than the unified scale $M_{u}$. Secondly, $\lambda^{\prime}$ must be fine-tuned by an amount $\Delta_{\lambda^{\prime}}$ to obtain the electroweak scale $v$ from the scale $v^{\prime}$. The total fine-tuning with Higgs Parity is the product

$$
\Delta_{H P}=\Delta_{m^{2}} \Delta_{\lambda^{\prime}}=\frac{v^{\prime 2}}{\Lambda^{2}} \times \frac{v^{2}}{{v^{\prime}}^{2}}=\frac{v^{2}}{\Lambda^{2}},
$$

which is independent of $v^{\prime}$. This is because a smaller $v^{\prime}$ requires more fine-tuning in $\Delta_{m^{2}}$, but this is compensated by less fine-tuning in $\Delta_{\lambda^{\prime}}$ to obtain the electroweak scale from the scale $v^{\prime}$. It is important to note that Higgs Parity, $H \leftrightarrow H^{\prime}$, ensures that the mass terms for $H$ and $H^{\prime}$ in (2.1) are identical, so that the single fine-tune by $\Delta_{m^{2}}$ protects both scalars to the scale $v^{\prime}$. Given that the SM Higgs must be protected for electroweak symmetry breaking, there is no additional cost to protect $H^{\prime}$ : the smallness of the scale $v^{\prime} \ll \Lambda$ requires no unnaturalness beyond that already needed for the weak scale. The total fine-tuning of the theory $\Delta_{H P}=v^{2} / \Lambda^{2}$ is nothing but the electroweak fine-tuning, which may be explained by environmental selection [41, 42].

This is in contrast to the usual $\mathrm{SO}(10)$ unification with an intermediate scale $v_{I}$. A smaller intermediate scale does not reduce the fine-tuning to obtain the electroweak scale, and hence the total fine-tuning is

$$
\Delta_{\mathrm{SO}(10)}=\frac{v_{I}^{2}}{\Lambda^{2}} \times \frac{v^{2}}{\Lambda^{2}}
$$

This extra fine-tuning $v_{I}^{2} / \Lambda^{2}$ cannot be explained by environmental selection of the electroweak scale, and requires an additional explanation.

\section{Gauge coupling unification and parity breaking scale}

We assume an $\mathrm{SO}(10)$ gauge symmetry at a high energy scale, broken to 3221 or 422 at the unification scale. These are then broken to the SM gauge group by the VEV of $H^{\prime}$. One possibility is that Higgs Parity is $C_{L R}$, a $Z_{2}$ subgroup of $\mathrm{SO}(10)$ that interchanges $\mathrm{SU}(2)_{L}$ 
and $\mathrm{SU}(2)_{R}$. In this case, the symmetry breaking chain and the required Higgs fields are

$$
\begin{array}{lllll}
\mathrm{SO}(10) & \stackrel{\phi_{210}}{\longrightarrow} & 3221 \times C_{L R} & \stackrel{H^{\prime}}{\longrightarrow} & \mathrm{SU}(3) \times \mathrm{SU}(2)_{L} \times \mathrm{U}(1)_{Y}, \\
\mathrm{SO}(10) & \stackrel{\phi_{54}}{\longrightarrow} & 422 \times C_{L R} & \stackrel{H^{\prime}}{\longrightarrow} & \mathrm{SU}(3) \times \mathrm{SU}(2)_{L} \times \mathrm{U}(1)_{Y} .
\end{array}
$$

To solve the strong $\mathrm{CP}$ problem, the symmetry to begin with is $\mathrm{SO}(10) \times C P$. This symmetry is broken by the VEV of a field that is odd under both $C_{L R}$ and $\mathrm{CP}$, so that the residual $Z_{2}$ symmetry for Higgs Parity is $C_{L R} * C P=P_{L R}$ and includes spacetime parity. In this case

$$
\begin{array}{lllll}
\mathrm{SO}(10) \times C P & \stackrel{\phi_{45}^{-}}{\longrightarrow} & 3221 \times P_{L R} & \stackrel{H^{\prime}}{\longrightarrow} & \mathrm{SU}(3) \times \mathrm{SU}(2)_{L} \times \mathrm{U}(1)_{Y}, \\
\mathrm{SO}(10) \times C P & \stackrel{\phi_{210}^{-}}{\longrightarrow} & 422 \times P_{L R} & \stackrel{H^{\prime}}{\longrightarrow} & \mathrm{SU}(3) \times \mathrm{SU}(2)_{L} \times \mathrm{U}(1)_{Y} .
\end{array}
$$

In this section we compute the running of the gauge coupling constants from IR to UV, treating the Higgs Parity symmetry breaking scale $v^{\prime}$ as a free parameter.

Values of the SM gauge couplings derived from experiment are

$$
g_{1}\left(m_{t}\right)=0.4626, g_{2}\left(m_{t}\right)=0.64779, g_{3}\left(m_{t}\right)=1.1666
$$

in the $\overline{\mathrm{MS}}$ scheme at a renormalization scale of $m_{t}$. Here the hypercharge coupling is given in the normalization appropriate for grand unification and is called $g_{1}$, or occasionally $g_{Y}$ to avoid confusion with the $B-L$ gauge coupling. Between the electroweak scale and the scale $v^{\prime}$, the RGE equation at the two-loop level is given by [43]

$$
\frac{\mathrm{d}}{\operatorname{dln} \mu}\left(\begin{array}{c}
\frac{2 \pi}{\alpha_{1}} \\
\frac{2 \pi}{\alpha_{2}} \\
\frac{2 \pi}{\alpha_{3}}
\end{array}\right)=\left(\begin{array}{c}
-\frac{41}{10} \\
\frac{19}{6} \\
7
\end{array}\right)+\left(\begin{array}{ccc}
-\frac{199}{100} & -\frac{27}{20} & -\frac{22}{5} \\
-\frac{9}{20} & -\frac{35}{12} & -6 \\
-\frac{11}{20} & -\frac{9}{4} & 13
\end{array}\right)\left(\begin{array}{c}
\frac{\alpha_{1}}{2 \pi} \\
\frac{\alpha_{2}}{2 \pi} \\
\frac{\alpha_{3}}{2 \pi}
\end{array}\right) .
$$

$$
\text { 4.1 } \mathrm{SU}(3) \times \mathrm{SU}(2) \times \mathrm{SU}(2) \times \mathrm{U}(1)
$$

We match the $\mathrm{SU}(3) \times \mathrm{SU}(2) \times \mathrm{U}(1)$ gauge coupling constants to those of 3221 at the $W^{\prime}$ mass fixed by Higgs Parity,

$$
\frac{2 \pi}{\alpha_{Y}\left(m_{W^{\prime}}\right)}=\left(\frac{2}{5}\right) \frac{2 \pi}{\alpha_{B-L}\left(m_{W^{\prime}}\right)}+\left(\frac{3}{5}\right) \frac{2 \pi}{\alpha_{2}\left(m_{W^{\prime}}\right)}-\frac{1}{10}, \quad m_{W^{\prime}}=\frac{g_{2}}{\sqrt{2}} v^{\prime},
$$

Since $W^{\prime}$ is the only heavy charged gauge boson at this scale, no mass-dependent threshold corrections are introduced from the gauge bosons. The RGE equation in the 3221 theory is $[43]$

$$
\frac{\mathrm{d}}{\mathrm{d} \ln \mu}\left(\begin{array}{c}
\frac{2 \pi}{\alpha_{1}} \\
\frac{2 \pi}{\alpha_{2}} \\
\frac{2 \pi}{\alpha_{3}}
\end{array}\right)=\left(\begin{array}{c}
-\frac{9}{2} \\
\frac{19}{6} \\
7
\end{array}\right)+\left(\begin{array}{lll}
-\frac{23}{8} & -\frac{27}{4} & -2 \\
-\frac{9}{8} & -\frac{35}{12} & -6 \\
-\frac{1}{4} & -\frac{9}{2} & 13
\end{array}\right)\left(\begin{array}{l}
\frac{\alpha_{1}}{2 \pi} \\
\frac{\alpha_{2}}{2 \pi} \\
\frac{\alpha_{3}}{2 \pi}
\end{array}\right) .
$$

Here we only show the contributions from gauge bosons, SM fermions, $H$ and $H^{\prime}$; contributions from $X$ states are shown in appendix A. 
We match the 3221 gauge couplings to that of $\mathrm{SO}(10)$ at the mass, $M_{X Y}$, of the XY gauge boson of charge $(3,2,2,-1 / 3)$. The only heavy gauge boson, other than the $X Y$ gauge boson, has 3221 quantum numbers $(3,1,1,2 / 3)$. Taking this gauge boson to have mass $r_{X Y} M_{X Y}$, gives threshold corrections

$$
\begin{array}{ll}
\frac{2 \pi}{\alpha_{1}\left(M_{X Y}\right)}=\frac{2 \pi}{\alpha_{10}\left(M_{X Y}\right)}+14 \ln r_{X Y}-\frac{4}{3}+\Delta_{1} & \equiv \frac{2 \pi}{\alpha_{10}\left(M_{X Y}\right)}+\Delta_{1, G}+\Delta_{1}, \\
\frac{2 \pi}{\alpha_{2}\left(M_{X Y}\right)}=\frac{2 \pi}{\alpha_{10}\left(M_{X Y}\right)}-1+\Delta_{2} & \equiv \frac{2 \pi}{\alpha_{10}\left(M_{X Y}\right)}+\Delta_{2, G}+\Delta_{2}, \\
\frac{2 \pi}{\alpha_{3}\left(M_{X Y}\right)}=\frac{2 \pi}{\alpha_{10}\left(M_{X Y}\right)}+\frac{7}{2} \ln r_{X Y}-\frac{5}{6}+\Delta_{3} & \equiv \frac{2 \pi}{\alpha_{10}\left(M_{X Y}\right)}+\Delta_{3, G}+\Delta_{3},
\end{array}
$$

where $\Delta_{i}$ denote possible threshold corrections from scalars and fermions. If the $\mathrm{SO}(10)$ symmetry is broken by a VEV of $\mathbf{4 5}, r_{X Y}=2$. If it is broken by the VEV of the SU(4) adjoint part of $\mathbf{2 1 0}, r_{X Y}=\sqrt{2}$. The VEV of $\mathbf{5 4}$ or the SU(4) singlet part of $\mathbf{2 1 0}$ gives a mass only to the $X Y$ gauge bosons, and makes $r_{X Y}$ smaller.

For each $M_{X Y}$, the threshold correction from scalars and fermions required for unification is

$$
\Delta \equiv \max _{i, j}\left|\left(\frac{2 \pi}{\alpha_{i}}-\Delta_{i, G}\right)-\left(\frac{2 \pi}{\alpha_{j}}-\Delta_{j, G}\right)\right|=\max _{i, j}\left|\Delta_{i}-\Delta_{j}\right|
$$

In figure 3 , we show contours of $\Delta$ in the $\left(v^{\prime}, M_{X Y}\right)$ plane, assuming $r_{X Y}=2$ (left) and $1 / 2$ (right). The dot indicates the point where $\Delta=0$. In the upper/lower panel, we assume that the $X$ multiplet generating the up yukawa couplings is $\mathbf{4 5 / 5 4}$. We fix the $X$ masses so that the quark yukawa couplings are reproduced for $x=1$. In the gray-shaded region, the Landau pole of the $\mathrm{SO}(10)$ gauge coupling is less than $10 M_{X Y}$, so that the precision of gauge coupling unification is spoiled. The blue-shaded region predicts too rapid a proton decay rate and is excluded by Super Kamiokande [44]. The blue dotted line shows the sensitivity of Hyper Kamiokande [45].

As $x$ is varied so the required value of $M_{X}$ changes. However, in the case that the entire $\mathrm{SO}(10)$ multiplet is degenerate, an order of magnitude change in $M_{X}$ only changes $\Delta$ by $\sim 1$, as this is a two loop effect. The different 3221 irreducible representations, $X_{a}$, within a single $\mathrm{SO}(10)$ multiplet receive non-degeneracies of only few $10 \%$ or less from gauge radiative corrections below $M_{X Y}$. However, for successful flavor physics we allow order unity tree-level splittings between these masses leading to contributions to $\Delta$ of $(4 / 3) C \ln \left(M_{a} / M_{b}\right)$, where $C$ is a quadratic Casimir, normalized to $1 / 2$ for a fundamental representation. Order unity splittings can give $\Delta \sim 1-3$, depending on the size and number of the $X$ multiplets.

In appendix B we compute contributions to $\Delta$ from scalar multiplets that break $\mathrm{SO}(10)$. $\Delta$ is typically smaller than 1 if $\mathbf{4 5}$ is the only such multiplet, while $\mathbf{5 4}$ and $\mathbf{2 1 0}$ multiplets allow for $\Delta$ of a few and 10 , respectively.

Higher dimensional interactions between the $\mathrm{SO}(10)$ symmetry breaking field and two field strengths of $\mathrm{SO}(10)$ gauge fields in general split the gauge coupling constants at the unification scale. Assuming a suppression scale of the reduced Planck mass, splittings from 

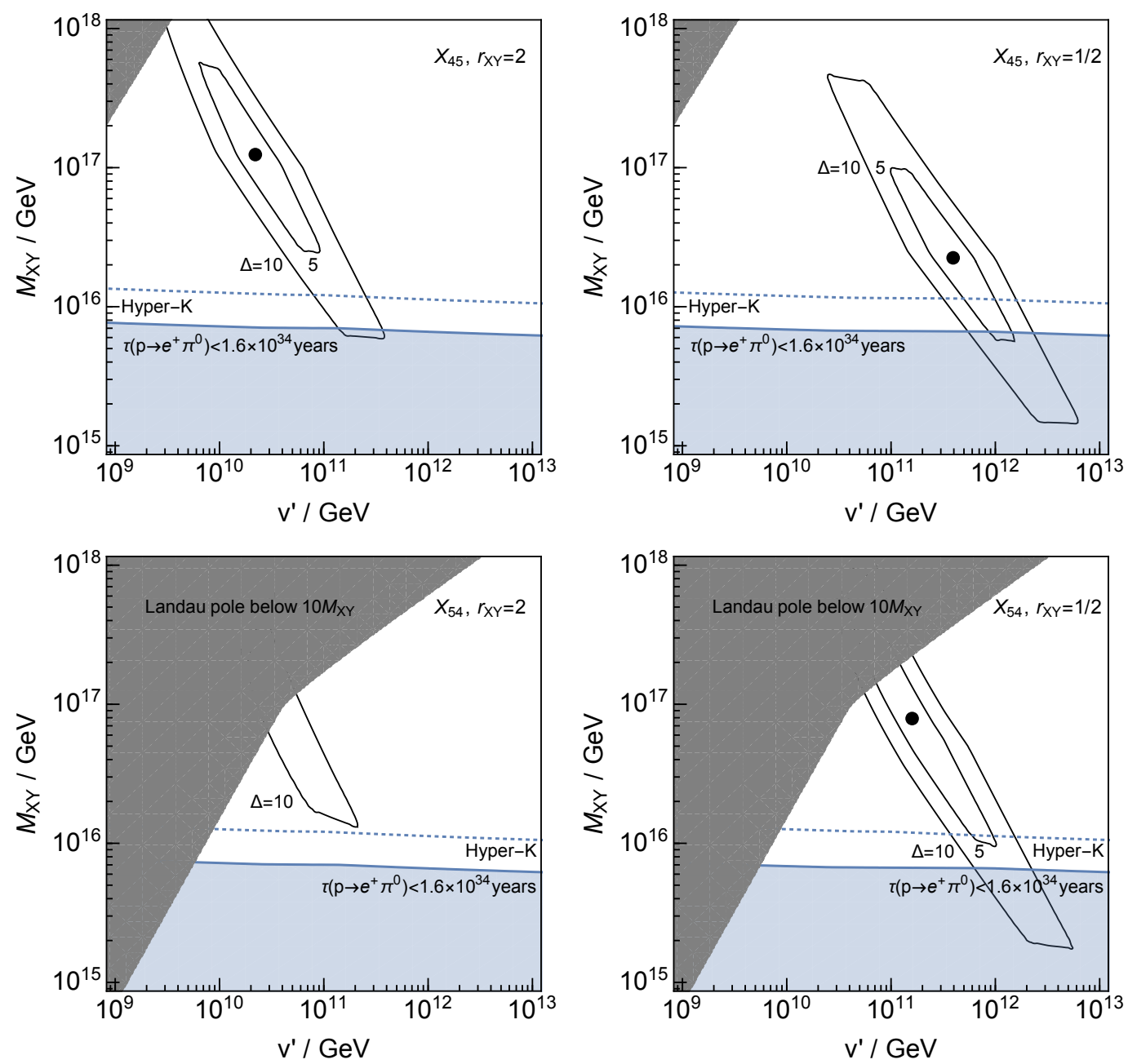

Figure 3. Determination of $v^{\prime}$ and $M_{X Y}$ in the 3221 theory from gauge coupling unification alone. The left (right) panels are for differing values of the unified gauge threshold corrections, and the contours show the effects of unified threshold corrections from scalars and fermions. The upper (lower) panels have the top yukawa coupling generated from the exchange of a 45 (54) $X$ state.

a dimension five operator typically give $\Delta \simeq 10$ for a unification scale of $10^{17} \mathrm{GeV}$. In theories with CP symmetry at the unification scale, which solve the strong CP problem, the dimension five operator is forbidden, and the splittings from a dimension six operator typically give $\Delta \simeq 1$. At lower values of the unification scale these values of $\Delta$ are reduced.

\section{$4.2 \quad \mathrm{SU}(4) \times \mathrm{SU}(2) \times \mathrm{SU}(2)$}

We match the SM gauge coupling constants to those of the 422 theory at the $W^{\prime}$ mass,

$$
\begin{aligned}
& \frac{2 \pi}{\alpha_{3}\left(m_{W^{\prime}}\right)}=\frac{2 \pi}{\alpha_{4}\left(m_{W^{\prime}}\right)}+\frac{7}{2} \ln \frac{g_{4}}{g_{2}}-\frac{1}{6}, \quad m_{W^{\prime}}=\frac{g_{2}}{\sqrt{2}} v^{\prime}, \\
& \frac{2 \pi}{\alpha_{1}\left(m_{W^{\prime}}\right)}=\left(\frac{2}{5}\right) \frac{2 \pi}{\alpha_{4}\left(m_{W^{\prime}}\right)}+\left(\frac{3}{5}\right) \frac{2 \pi}{\alpha_{2}\left(m_{W^{\prime}}\right)}+\frac{28}{5} \ln \frac{g_{4}}{g_{2}}-\frac{7}{15} .
\end{aligned}
$$




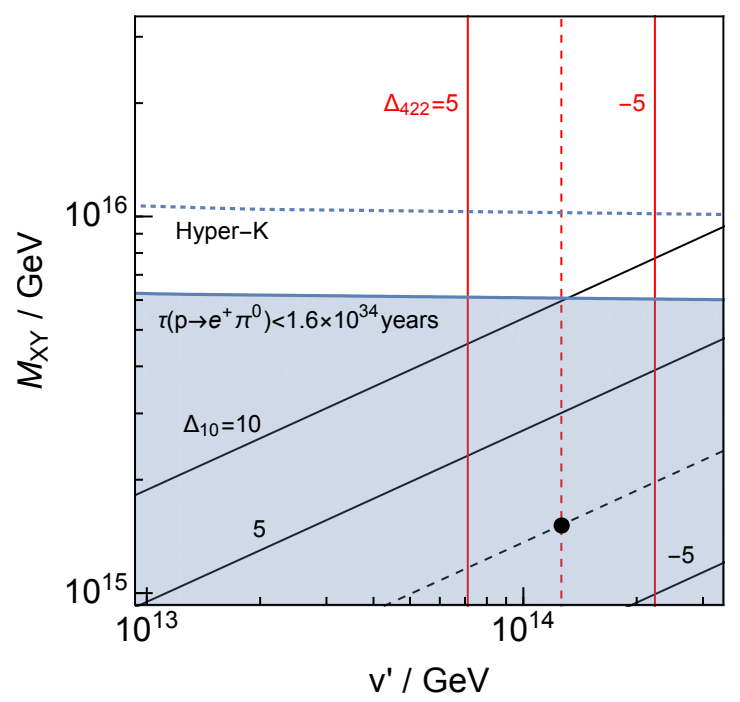

Figure 4. Determination of $v^{\prime}$ and $M_{X Y}$ in the 422 theory from gauge coupling unification alone. Contours of threshold corrections from scalars and fermions at the scale $v^{\prime}\left(\Delta_{422}\right)$ and at the scale $M_{X Y}\left(\Delta_{10}\right)$ are shown in red and black.

Since the values of $\alpha_{4}$ and $\alpha_{2}$ are known, the successful embedding of $\mathrm{U}(1)_{Y}$ into the PatiSalam gauge group fixes the scale $v^{\prime}$. To take into account a possible threshold correction, we define

$$
\Delta_{422} \equiv \frac{2 \pi}{\alpha_{1}\left(m_{W^{\prime}}\right)}-\frac{2}{5} \frac{2 \pi}{\alpha_{3}\left(m_{W^{\prime}}\right)}-\frac{3}{5} \frac{2 \pi}{\alpha_{2}\left(m_{W^{\prime}}\right)}-\frac{21}{5} \ln \frac{g_{4}}{g_{2}}+\frac{2}{5} .
$$

The RGE equation of the $\mathrm{SU}(4) \times \mathrm{SU}(2) \times \mathrm{SU}(2)$ gauge coupling constants is [43]

$$
\frac{\mathrm{d}}{\mathrm{d} \ln \mu}\left(\begin{array}{c}
\frac{2 \pi}{\alpha_{2}} \\
\frac{2 \pi}{\alpha_{4}}
\end{array}\right)=\left(\begin{array}{c}
\frac{8}{3} \\
10
\end{array}\right)+\left(\begin{array}{cc}
-\frac{37}{6} & -\frac{75}{4} \\
-\frac{15}{2} & \frac{117}{4}
\end{array}\right)\left(\begin{array}{l}
\frac{\alpha_{2}}{2 \pi} \\
\frac{\alpha_{4}}{2 \pi}
\end{array}\right) .
$$

Here we only show the contribution from the gauge bosons, the SM fermions, $H$ and $H^{\prime}$. The contribution from the $X$ states is shown in appendix A.

We match the 422 gauge couplings at the mass, $M_{X Y}$, of the XY gauge boson, which is the only heavy gauge boson. The threshold corrections at $M_{X Y}$ are

$$
\begin{array}{ll}
\frac{2 \pi}{\alpha_{2}\left(M_{X Y}\right)}=\frac{2 \pi}{\alpha_{10}\left(M_{X Y}\right)}-1+\Delta_{2} & \equiv \frac{2 \pi}{\alpha_{10}\left(M_{X Y}\right)}+\Delta_{2, G}+\Delta_{2}, \\
\frac{2 \pi}{\alpha_{4}\left(M_{X Y}\right)}=\frac{2 \pi}{\alpha_{10}\left(M_{X Y}\right)}-\frac{2}{3}+\Delta_{4} & \equiv \frac{2 \pi}{\alpha_{10}\left(M_{X Y}\right)}+\Delta_{4, G}+\Delta_{4},
\end{array}
$$

where $\Delta_{4,2}$ denote possible threshold corrections from scalars and fermions. For each $M_{X Y}$, we quantify the required value of the threshold correction by

$$
\Delta_{10} \equiv\left(\frac{2 \pi}{\alpha_{4}}-\Delta_{4, G}\right)-\left(\frac{2 \pi}{\alpha_{2}}-\Delta_{2, G}\right)=\Delta_{4}-\Delta_{2}
$$

In figure 4 , we show the contours of $\Delta_{422}$ and $\Delta_{10}$. The parameter point where no threshold correction is required is already excluded by Super Kamiokande. A threshold correction 
of $\Delta_{10} \sim 10$ is necessary to evade the bound from proton decay. We estimate the typical magnitude of the threshold corrections from the unified scalar multiplets that break $\mathrm{SO}(10)$ belonging to $\mathbf{5 4}$ or $\mathbf{2 1 0}$ in appendix $\mathrm{B}$, and show that $\Delta_{10}$ is typically $O(1)$. This is because of the smallness of the contribution of scalar particles to the renormalization of gauge couplings. Threshold correction can be large if the theory near the unification scale is nonminimal; if the unified scale arises from the supersymmetry breaking scale, the threshold correction can be easily as large as 10 .

\section{Yukawa couplings}

The predictions from Higgs Parity coupling unification are affected by threshold corrections to $\lambda_{\mathrm{SM}}\left(v^{\prime}\right)$. The SM yukawa couplings are generated from the mixing of $(q, \bar{q}, \ell, \bar{\ell})$ with the $X$ states when parity is broken by $H^{\prime}=v^{\prime}$. The leading correction to $\lambda_{\mathrm{SM}}\left(v^{\prime}\right)$ is expected to arise from the generation of the top quark yukawa coupling. In this section we discuss how the SM yukawa couplings arise from the $\mathrm{SO}(10)$ unified theory via interactions of (3.3). We also show that there is a simple understanding of why the $b / \tau$ mass ratio deviates from the simplest expectation from grand unification, as well as why the neutrino masses and the mixings are not as hierarchical as those of quarks. We also comment on a possible impact on leptogenesis [46].

The $X$ states arise from $\mathbf{4 5 , 5 4}$ or $\mathbf{1 0}$ representations of $\mathrm{SO}(10)$, whose decomposition into 3221 is shown in table 2. $\mathbf{4 5}$ and $\mathbf{5 4}$ give up-type yukawa couplings and neutrino masses, while 10 gives down-type quark and charged lepton yukawa couplings. We do not consider larger representations as they lead to the gauge couplings blowing-up below the unification scale. For complex 3221 representations, $Q, U$ and $D$, we omit their complex conjugates, $\bar{Q}, \bar{U}$ and $\bar{D}$ from the table. Non-singlet $\mathrm{SU}(2)_{R}$ multiplets are decomposed into SM multiplets by giving the $\mathrm{U}(1)_{Y}$ charge as a subscript; thus $Q$, which is an $\mathrm{SU}(2)_{R}$ doublet, contains SM multiplets $\left(Q_{1 / 6}, Q_{-5 / 6}\right)$.

Terms in the Lagrangian of the $\mathrm{SO}(10)$ theory that lead to quark and lepton masses are

$$
\mathcal{L}_{\mathrm{SO}(10)}=\left(\psi x_{45,54} X_{45,54}\right) \phi^{\dagger} \mathcal{O}_{G}+\frac{m_{45,54}}{2} X_{45,54}^{2} \mathcal{O}_{G}+\left(\psi x_{10} X_{10}\right) \phi \mathcal{O}_{G}+\frac{m_{10}}{2} X_{10}^{2} \mathcal{O}_{G}
$$

where $\psi(q, \ell, \bar{q}, \bar{\ell})$ and $\phi \supset H, H^{\prime}$ are both in $\mathbf{1 6}$, and $\mathcal{O}_{G}$ denotes possible insertions of fields with $\mathrm{SO}(10)$ symmetry breaking vevs. Note $\psi X_{54} \phi^{\dagger}$ is not an $\mathrm{SO}(10)$ invariant, and hence requires a non-trivial $\mathcal{O}_{G}$. In the following we analyze the yukawa couplings in the 3221 theory. The discussion for the 422 theory is almost the same, as the 422 symmetry does not impose relations between the parameters in the 3221 theory except for one case that we mention below. We study the generation of yukawa couplings in the up, down, charged lepton and neutrino sectors by integrating out the $X$ states.

\section{$5.1 \quad$ Up-type quark yukawa couplings}

The $X$ states for the up-type yukawas couplings are in $\mathbf{4 5}$ or $\mathbf{5 4}$. For $\mathbf{5 4}$, the up yukawa couplings arise from the interaction and mass term

$$
\mathcal{L}_{54_{Q}}=\bar{q} x_{Q} Q H^{\dagger}+q x_{Q} \bar{Q} H^{\prime \dagger}+m_{Q} Q \bar{Q}+\text { h.c. }
$$




\begin{tabular}{|c|c|c|c|c|c|c|c|c|c|c|c|c|c|c|c|}
\hline $\mathrm{SO}(10)$ & \multicolumn{5}{|c|}{54} & $\mathrm{SO}(10)$ & \multicolumn{6}{|c|}{45} & $\mathrm{SO}(10)$ & \multicolumn{2}{|c|}{10} \\
\hline & $Q$ & & & & $S$ & & $Q$ & $U$ & & $T_{L}$ & $T_{R}$ & $S$ & & $D$ & $\Delta$ \\
\hline $\mathrm{SU}(3)$ & 3 & 6 & 8 & 1 & 1 & $\mathrm{SU}(3)$ & 3 & 3 & 8 & 1 & 1 & 1 & $\mathrm{SU}(3)$ & 3 & 1 \\
\hline $\mathrm{SU}(2)$ & 2 & 1 & 1 & 3 & 1 & $\mathrm{SU}(2)$ & 2 & 1 & 1 & 3 & 1 & 1 & $\mathrm{SU}(2)$ & 1 & 2 \\
\hline $\mathrm{SU}(2)$ & 2 & 1 & 1 & 3 & 1 & $\mathrm{SU}(2)$ & 2 & 1 & 1 & 1 & 3 & 1 & $\mathrm{SU}(2)$ & 1 & 2 \\
\hline $\mathrm{U}(1)$ & $-1 / 3$ & $-2 / 3$ & 0 & 0 & 0 & $\mathrm{U}(1)$ & $-1 / 3$ & $2 / 3$ & 0 & 0 & 0 & 0 & $\mathrm{U}(1)$ & $-1 / 3$ & 0 \\
\hline
\end{tabular}

Table 2. Decomposition of $X$ states into representations of 3221 . For complex representations, complex conjugations of them are understood.

Note that below the $\mathrm{SO}(10)$ breaking scale, the $\psi(Q, U, \ldots) \phi$ couplings $x_{X}$ and masses $m_{X}$ are given for each 3221 component of $X:(Q, U, D, \ldots)$. We allow these couplings and masses to deviate from strict $\mathrm{SO}(10)$ relations by order unity amounts via $\mathcal{O}_{G}$.

Here and below we neglect flavor mixing, which can be straightforwardly taken into account, so that $x_{Q}$ and $m_{Q}$ are real parameters referring to a single generation. We show how the SM up-type yukawa coupling arises in the upper most panel of figure 5. Because of the non-zero $\left\langle H^{\prime}\right\rangle, Q_{1 / 6}$ and $q$ mix with each other. The mixings in figure 5 are given by

$$
s_{X} \equiv \sin \theta_{X}=\frac{x_{X} v^{\prime}}{\sqrt{m_{X}^{2}+x_{X}^{2} v^{\prime 2}}} .
$$

A linear combination of $Q_{1 / 6}$ and $q$ obtains a mass $\sqrt{m_{Q}^{2}+x_{Q}^{2} v^{\prime 2}}$, paired with $\bar{Q}_{-1 / 6}$. The orthogonal linear combination of $Q_{1 / 6}$ and $q$ becomes a doublet quark of the SM acquiring a yukawa coupling to $\bar{q}_{-2 / 3}$, a right-handed up-type quark, of

$$
y_{u}=x_{Q} s_{Q}=\frac{x_{Q}^{2} v^{\prime}}{\sqrt{m_{Q}^{2}+x_{Q}^{2} v^{\prime 2}}} .
$$

Except for the top yukawa coupling, we expect $m_{Q} \gg x_{Q} v^{\prime}$ to be a good approximation, so that $y_{u} \simeq x_{Q}^{2} v^{\prime} / m_{Q}$ for the up and charm quarks. The $O(1)$ top yukawa coupling requires $m_{Q} \lesssim x_{Q} v^{\prime} . Q_{-5 / 6}$ and $\bar{Q}_{5 / 6}$ obtain a mass $m_{Q}$.

When the $X$ states arise from $\mathbf{4 5}$, we have

$$
\mathcal{L}_{45_{Q U}}=\bar{q} x_{Q} Q H^{\dagger}+q x_{Q} \bar{Q} H^{\prime \dagger}+m_{Q} Q \bar{Q}+q x_{U} \bar{U} H^{\dagger}+\bar{q} x_{U} U H^{\prime \dagger}+m_{U} U \bar{U}+\text { h.c. }
$$

The fate of $Q \bar{Q}$ and $q$ are the same as for 54. A linear combination of $\bar{q}_{-2 / 3}$ and $\bar{U}$ pairs with $U$ and obtains a mass $\sqrt{m_{U}^{2}+x_{U}^{2} v^{\prime 2}}$. The orthogonal combination becomes a $\bar{u}$ of the SM, so that the corresponding up-type yukawa coupling is

$$
y_{u}=x_{Q} s_{Q} c_{U}+x_{U} c_{Q} s_{U}=\frac{\left(x_{Q}^{2} m_{U}+x_{U}^{2} m_{Q}\right) v^{\prime}}{\sqrt{m_{U}^{2}+x_{U}^{2}{v^{\prime}}^{2}} \sqrt{m_{Q}^{2}+x_{Q}^{2} v^{\prime 2}}} .
$$

Small up and charm yukawa couplings are explained by $m_{Q, U} \gg x_{Q, U} v^{\prime}$ or $m_{Q, U} \ll x_{Q, U} v^{\prime}$. 


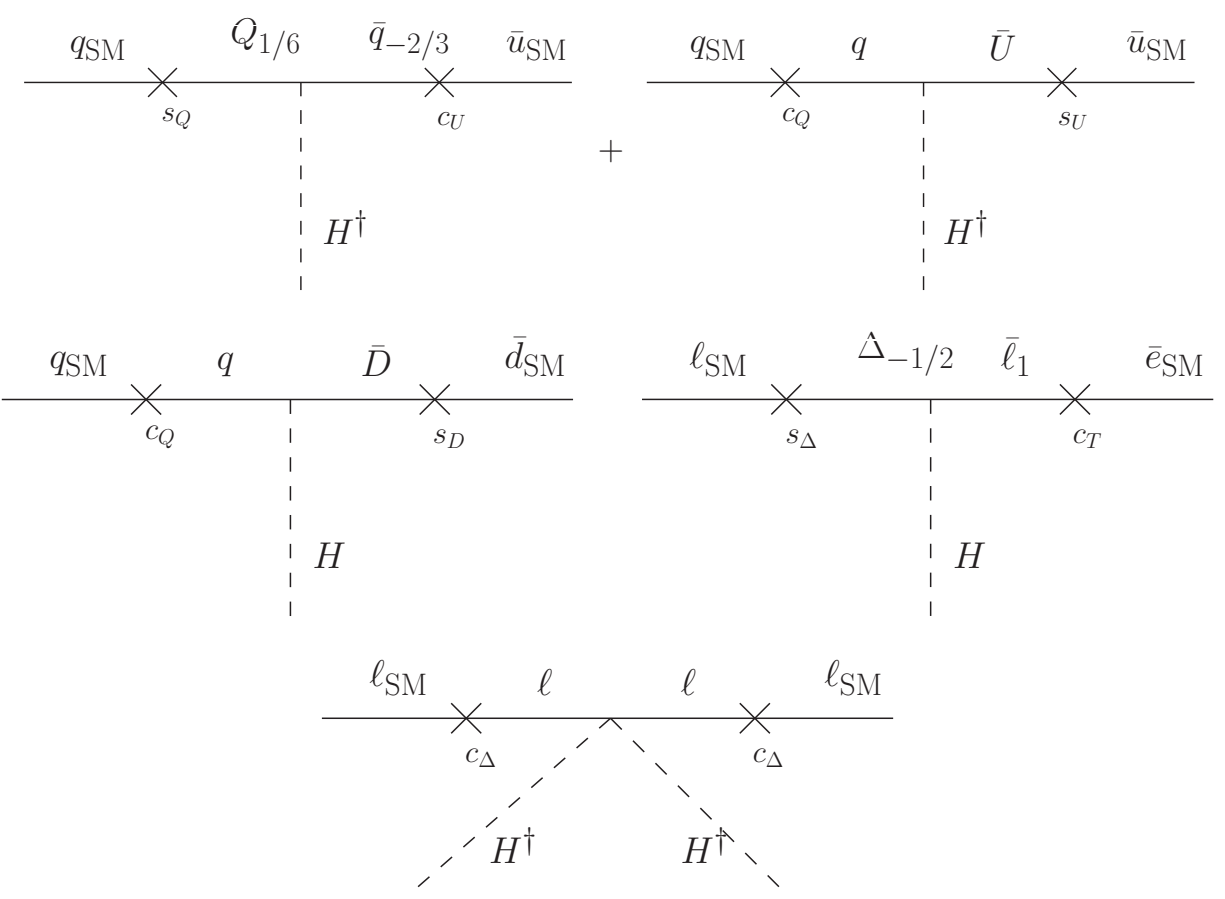

Figure 5. The generation of SM fermion masses. The mixing angles are defined so that they vanish in the limit $m_{X} \gg x_{X} v^{\prime}, s_{X}=x_{X} v^{\prime} / \sqrt{m_{X}^{2}+x_{X}^{2} v^{\prime 2}}$.

\subsection{Down-type quark yukawa coupling}

The $X$ states for down-type quark yukawa couplings are in $\mathbf{1 0}$ of $\mathrm{SO}(10)$, as larger representations result in non-perturbative gauge couplings. Yukawa couplings arise from

$$
\mathcal{L}_{10_{D}}=q x_{D} \bar{D} H+\bar{q} x_{D} D H^{\prime}+m_{D} D \bar{D}+\text { h.c. }
$$

A linear combination of $\bar{q}_{1 / 3}$ and $\bar{D}$ obtains a mass $\sqrt{m_{D}^{2}+x_{D}^{2} v^{\prime 2}}$, paired with $D$. The orthogonal linear combination is the SM right-handed down quark. The SM down-type yukawa coupling is

$$
y_{d}=x_{D} c_{Q} s_{D}=\frac{x_{D}^{2} v^{\prime}}{\sqrt{m_{D}^{2}+x_{D}^{2} v^{\prime 2}}} \frac{m_{Q}}{\sqrt{m_{Q}^{2}+x_{Q}^{2} v^{\prime 2}}} .
$$

\subsection{Charged lepton yukawa couplings}

The $X$ states for charged lepton yukawa couplings are also in $\mathbf{1 0}$ of $\mathrm{SO}(10)$, and the yukawa couplings arise from

$$
\mathcal{L}_{10_{\Delta}}=\bar{\ell} x_{\Delta} \Delta H+\ell x_{\Delta} \Delta H^{\prime}+\frac{1}{2} m_{\Delta} \Delta^{2}+\text { h.c. }
$$

A linear combination of $\Delta_{-1 / 2}$ and $\ell$ obtains a mass $\sqrt{m_{\Delta}^{2}+x_{\Delta}^{2} v^{\prime 2}}$, paired with $\Delta_{1 / 2}$. The orthogonal linear combination is the SM lepton doublet. 
The SM charged lepton yukawa couplings depend on whether the $X$ states for the up-type quark is $\mathbf{5 4}$ or $\mathbf{4 5}$. If it is $\mathbf{5 4}, \bar{\ell}_{1}$ is the SM right-handed charged lepton. If it is 45, we need to take into account the following interaction,

$$
\mathcal{L}_{45_{T}}=\ell x_{T} T_{L} H^{\dagger}+\bar{\ell} x_{T} T_{R} H^{\prime \dagger}+\frac{1}{2} m_{T} T_{L}^{2}+\frac{1}{2} m_{T} T_{R}^{2}+\text { h.c. }
$$

A linear combination of $\bar{\ell}_{1}$ and $T_{R, 1}$ obtains a mass $\sqrt{m_{T}^{2}+x_{T}^{2} v^{\prime 2}}$, paired with $T_{R,-1}$. The orthogonal linear combination is the SM right-handed charged lepton. The SM charged lepton yukawa coupling is

$$
y_{e}=x_{\Delta} s_{\Delta} \times\left\{\begin{array}{ll}
1 \\
c_{T}
\end{array}=\frac{x_{\Delta}^{2} v^{\prime}}{\sqrt{m_{\Delta}^{2}+x_{\Delta}^{2} v^{\prime 2}}} \times\left\{\begin{array}{ll}
1 & : X_{54} \\
\frac{m_{T}}{\sqrt{m_{T}^{2}+x_{T}^{2} v^{\prime 2}}} & : X_{45}
\end{array} .\right.\right.
$$

\subsection{Neutrino masses and mixing}

If the $X$ states of up-type quarks are $\mathbf{5 4}$, neutrino masses may arise from the interactions and mass term,

$$
\mathcal{L}_{54_{S}}=\ell x_{S} S H^{\dagger}+\bar{\ell} x_{S} S H^{\prime \dagger}+\frac{1}{2} m_{S} S^{2}+\text { h.c. }
$$

In the 422 theory $m_{S}=m_{U}$ and $x_{S}=\sqrt{3} / 2 x_{U}$. At tree-level, only one linear combination of $\nu$ and $N$, which is predominantly $N$, obtains a mass, and the SM neutrino remains massless. However, since lepton and chiral symmetries are broken by $m_{S}$, there is no symmetry forbidding the seesaw operator $\left(\ell H^{\dagger}\right)^{2}$ at the bottom of figure 5 which should arise from quantum corrections. Taking into account mixing between $\ell$ and $\Delta_{-1 / 2}$, the neutrino mass is

$$
m_{\nu} \sim \frac{1}{16 \pi^{2}} \frac{x_{S}^{2} v^{2}}{m_{S}} c_{\Delta}^{2}=\frac{1}{16 \pi^{2}} \frac{x_{S}^{2} v^{2}}{m_{S}} \frac{m_{\Delta}^{2}}{m_{\Delta}^{2}+x_{\Delta}^{2} v^{\prime 2}}
$$

If the $X$ states of up-type quarks are 45, the neutrino mass arises from eq. (5.10), where the exchange of $T_{L}$ generates the operators $\left(\ell H^{\dagger}\right)^{2}$,

$$
m_{\nu}=\frac{x_{T}^{2} v^{2}}{m_{T}} \frac{m_{\Delta}^{2}}{m_{\Delta}^{2}+x_{\Delta}^{2} v^{\prime 2}}
$$

Next we consider aspects of flavor mixing. Although the same $\mathrm{SO}(10)$ states, $X_{45,54}$, contribute to both up-type quark and neutrino masses, the lack of mass hierarchies and large mixing angles of neutrinos compared with up-type quarks can be understood. Assuming $m_{\Delta} \ll x_{\Delta} v^{\prime}$ only for the third generation, the neutrino mass matrix is given by

$$
m_{\nu} \sim\left(\frac{1}{16 \pi^{2}}\right)_{54} \frac{v^{2}}{v^{\prime}}\left(\begin{array}{ccc}
1 & & \\
& 1 & \\
& & c_{\Delta_{3}}
\end{array}\right) y_{u}\left(\begin{array}{ccc}
1 & & \\
& 1 & \\
& & c_{\Delta_{3}}
\end{array}\right), \quad c_{\Delta_{3}}=\frac{m_{\Delta_{3}}}{\sqrt{m_{\Delta_{3}}^{2}+x_{\Delta_{3}}^{2} v^{\prime 2}}} \ll 1
$$


where the factor of $1 / 16 \pi^{2}$ applies only for $X_{54}$. With $c_{\Delta_{3}}=O\left(10^{-2}\right)$, the neutrino mass matrix is not near-diagonal nor hierarchical, except for the $(1,1)$ component. Thus, in Higgs Parity Unification we are able to derive an order-of-magnitude mass relation

$$
m_{\nu_{2,3}} \sim\left(\frac{1}{16 \pi^{2}}\right)_{54} \frac{v}{v^{\prime}} m_{c}
$$

which is successful since the Higgs mass and coupling unification require $v^{\prime}=10^{10-12} \mathrm{GeV}$. The small up quark mass arises from the $(1,1)$ component of $(5.15)$, so that the lightest neutrino is much lighter than the other two neutrinos, giving a normal hierarchy with

$$
\frac{m_{\nu_{1}}}{m_{\nu_{2,3}}} \sim \frac{m_{u}}{m_{c}}
$$

Because of the suppression of the neutrino mass by $c_{\Delta_{3}}$, for a given mass $m_{S, T}$ the couplings $x_{S, T}$ are larger than expected from the usual see-saw relation $m_{\nu} \sim x_{S, T}^{2} v^{2} / m_{S, T}$. We expect that the lepton asymmetry produced by decays of $S$ and $T$ is enhanced, reducing the minimal reheating temperature for successful leptogenesis, whether thermal $[47,48]$ or non-thermal [49].

\subsection{A simple $\mathrm{SO}(10)$ theory of flavor}

The following renormalizable $\mathrm{SO}(10)$ model can economically describe all quark and lepton masses

$$
\begin{aligned}
\mathcal{L}= & \psi_{16} x_{45} X_{45} \phi_{16}^{\dagger}+\frac{1}{2} X_{45}\left(m_{45}+h_{45} \Sigma\right) X_{45} \\
& +\psi_{16} x_{10} X_{10} \phi_{16}+\frac{1}{2} X_{10}\left(m_{10}+h_{10} \Sigma\right) X_{10}+\text { h.c. }
\end{aligned}
$$

Here we introduce three generations of fermions, $\psi_{16}, X_{45}$ and $X_{10}$, with generation indices understood. The Higgs $H$ and $H^{\prime}$ are embedded into $\phi_{16} . \quad \Sigma$ is an $\mathrm{SO}(10)$ symmetry breaking field, and $x, m$ and $h$ are constants. Since $\Sigma$ does not appear in the above yukawa interactions, this model predicts $x_{Q}=x_{U}=x_{T}=2 / \sqrt{3} x_{S}$ and $x_{D}=x_{\Delta}$ at the $\mathrm{SO}(10)$ scale. However, while mass parameters $m_{X}$ are not necessarily unified, we assume that differing 3221 multiplets in the same $X$ representation have masses that are not hierarchically different from each other (e.g. $m_{D} \approx m_{\Delta}$ ).

Despite the unification of $x_{X}$, and departures from unification of $m_{X}$ by only $O(1)$ amounts, the neutrino masses and mixings can be obtained via $c_{\Delta_{3}}=O\left(10^{-2}\right)$, as explained in the previous sub-section. This requires that both $s_{\Delta_{3}}$ and $s_{D_{3}}$ are very close to unity, and hence $y_{b}=x_{D} c_{Q}$ and $y_{\tau}=x_{\Delta} c_{T}$. Thus $m_{b} / m_{\tau}$ differs from that predicted in minimal $\mathrm{SU}(5)$ unification schemes by $c_{Q} / c_{T}$, which arises from an $\mathrm{O}(1)$ difference between $m_{Q}$ and $m_{T}$. The ratios $m_{d} / m_{e}$ and $m_{s} / m_{\mu}$ can also be explained by $m_{D} / m_{\Delta}$ ratios at the $\mathrm{SO}(10)$ scale that are not far from unity.

The strong CP problem is solved by Left-Right symmetry including space-time parity. Above the $\mathrm{SO}(10)$ breaking scale, the symmetry of the theory is $\mathrm{SO}(10) \times C P$. This symmetry is broken down to Left-Right symmetry with space-time parity by the VEV of a field that is both Left-Right and CP odd. The CP symmetry requires that $x_{10,45}$ and $m_{10,45}$ 
are real in a certain field basis. When $\Sigma$ is made from an odd number of the Left-Right and CP odd fields, the couplings $h_{10,45}$ are pure-imaginary, explaining the CKM phase. In fact, one can check that the imaginary part can appear in any components of the SM yukawa couplings by expanding them in $h$ around the diagonal components of $x$ and $m_{X}$.

Ref. [18] shows that the quantum corrections to the strong CP phase arise at two-loop level. The corrections are shown to be below the current limit from the neutron electric dipole moment under the assumption that the couplings $x$ are $O(1)$ and $m_{X}$ are above $v^{\prime}$. This assumption is not valid for the $(3,3)$ components of $x_{D} \sim y_{b}$ and $m_{D} \sim 10^{-2} y_{b} v^{\prime}$. As a result, the suppression of the corrections found in [18] is not guaranteed, and the corrections may be as large as $10^{-6}$. We expect that the corrections are suppressed by an appropriate flavor structure of $x$ and $m_{X}$, which we leave to future work.

\subsection{The bottom-tau mass ratio and $x_{Q}$}

If the top quark mass is generated by $X_{54}$, then the bottom quark but not the tau, receives a suppression from the up-type quark yukawa sector; thus from eqs. (5.8) and (5.11) $y_{b}=$ $c_{Q} x_{D} s_{D}$ while $y_{\tau}=x_{\Delta} s_{\Delta}$. Assuming $x_{D}=x_{\Delta}$ at the unification scale, as well as $c_{\Delta, D} \ll 1$ to obtain the neutrino masses and mixing, we find $y_{b} / y_{\tau}=c_{Q}$ at the unified scale, which is renormalized to

$$
\frac{y_{b}}{y_{\tau}}\left(m_{Z}\right)=2.1 \frac{m_{Q}}{\sqrt{m_{Q}^{2}+x_{Q}^{2} v^{\prime 2}}} .
$$

To obtain the observed ratio of 1.6 , we need

$$
x_{Q} \simeq 1.5 y_{t} .
$$

If the top quark mass is generated by $X_{45}$, the tau yukawa coupling may be also suppressed by $c_{T}<1$. To obtain the bottom/tau ratio, $m_{T}>m_{Q}$ is required. Unless $m_{T} \simeq m_{Q}$, we may neglect the suppression of the tau yukawa coupling. Assuming $x_{D}=x_{\Delta}$ at the unification scale as well as $c_{\Delta, D} \ll 1$, the observed value of $m_{b} / m_{\tau}$ then requires

$$
x_{Q} \simeq 1.01 y_{t}
$$

where we assume $x_{Q} \simeq x_{U}$ and $m_{Q} \simeq m_{U}$.

The values of $x_{Q}$ from (5.20) and (5.21) are used to evaluate the top quark threshold correction to $\lambda_{\mathrm{SM}}\left(v^{\prime}\right)$ in section 6 .

\section{$6 \quad$ Prediction for the scale of parity breaking}

In section 2, we showed that the SM Higgs quartic coupling essentially vanishes at tree level at the Higgs Parity symmetry breaking scale, $v^{\prime}$. In this section, we compute threshold corrections to the Higgs quartic coupling and derive $v^{\prime}$ in terms of SM parameters.

\subsection{Threshold corrections to the SM quartic coupling}

The tree-level scalar potential is

$$
V_{\text {tree }}=\lambda\left(|H|^{2}+\left|H^{\prime}\right|^{2}\right)^{2}+\lambda^{\prime}|H|^{2}\left|H^{\prime}\right|^{2}-m^{2}\left(|H|^{2}+\left|H^{\prime}\right|^{2}\right) .
$$

After taking into account quantum corrections, the coupling $\lambda^{\prime}\left(v^{\prime}\right)$ becomes non-zero. 


\subsubsection{Threshold correction from charged gauge bosons}

The one-loop Coleman-Weinberg (CW) potential [50] from $W$ and $W^{\prime}$ bosons is

$$
V_{1-\text { loop }}=c|H|^{4} \ln \frac{|H|}{M}+c\left|H^{\prime}\right|^{4} \ln \frac{\left|H^{\prime}\right|}{M}, \quad c \equiv \frac{3}{64 \pi^{2}} g^{4},
$$

where $M$ is an arbitrary scale. A change of $M$ can be absorbed by a change of $\lambda$. The vev of $H^{\prime}$ satisfies

$$
m^{2}=2 \lambda v^{\prime 2}\left(1+\frac{c}{4 \lambda}+\frac{c}{2 \lambda} \ln \frac{v^{\prime 2}}{M^{2}}\right)
$$

After integrating out $H^{\prime}$, the potential for $H$, to leading order in $c$ and $\lambda^{\prime}$, is given by

$$
V(H) \simeq v^{\prime 2}\left(\lambda^{\prime}-\frac{c}{2}-c \ln \frac{v^{\prime 2}}{M^{2}}\right)|H|^{2}+\left(-\lambda^{\prime}+\frac{3}{4} c+c \ln \frac{v^{\prime 2}}{M^{2}}+c \ln \frac{|H|}{v^{\prime}}\right)|H|^{4} .
$$

To obtain the electroweak scale much smaller than $v^{\prime}$ requires $\lambda^{\prime} \simeq c / 2+2 c \ln \left(v^{\prime} / M\right)$, giving

$$
V(H) /|H|^{4} \simeq \frac{c}{4}\left(1+4 \ln \frac{|H|}{v^{\prime}}\right) .
$$

We match this potential to the one-loop CW potential of the SM from the $W$ boson,

$$
V_{\mathrm{SM}}(H) /|H|^{4}=\lambda_{\mathrm{SM}}(\mu)+\frac{3}{128 \pi^{2}} g^{4}\left(\ln \frac{g^{2}|H|^{2} / 2}{\mu^{2}}-\frac{3}{2}\right),
$$

where we take the $\overline{\mathrm{MS}}$ scheme. By matching $V_{\mathrm{SM}}(H)$ to $V(H)$ with $\mu=v^{\prime}$, we obtain

$$
\lambda_{\mathrm{SM}, W}\left(v^{\prime}\right) \simeq \frac{3}{64 \pi^{2}} g^{4} \ln \frac{e}{g / \sqrt{2}} .
$$

To suppress higher order corrections, the coupling $g$ should be evaluated around $v^{\prime}$.

\subsubsection{Threshold correction from neutral gauge bosons}

The threshold correction from $Z$ and $Z^{\prime}$ bosons can be estimated in a similar manner. After integrating out $H^{\prime}$ and fine-tuning for the electroweak scale, the Higgs potential is

$$
V(H) /|H|^{4} \simeq \frac{3\left(g^{2}+g^{\prime 2}\right)^{2}}{512 \pi^{2}}\left(1+4 \ln \frac{|H|}{v^{\prime}}-2 \ln \frac{g^{4}}{g^{4}-g^{\prime 4}}\right) .
$$

The one-loop CW potential of the SM from the $Z$ boson is

$$
V_{\mathrm{SM}}(H) /|H|^{4}=\lambda_{\mathrm{SM}}(\mu)+\frac{3}{256 \pi^{2}}\left(g^{2}+g^{\prime 2}\right)^{2}\left(\ln \frac{\left(g^{2}+g^{\prime 2}\right)|H|^{2} / 2}{\mu^{2}}-\frac{3}{2}\right) .
$$

Matching these results at $v^{\prime}$ gives the threshold correction

$$
\lambda_{\mathrm{SM}, Z}\left(v^{\prime}\right) \simeq \frac{3}{256 \pi^{2}}\left(g^{2}+{g^{\prime}}^{2}\right)^{2}\left(\ln \frac{e^{2}}{\left(g^{2}+g^{\prime 2}\right) / 2}-\ln \frac{g^{4}}{g^{4}-g^{\prime 4}}\right) .
$$




\subsubsection{Threshold correction from top quarks}

The threshold correction from top quarks is model-dependent. Let us first consider the case where the $X$ state for the top quark is $\mathbf{5 4}$, where the relevant interaction is shown in eq. (5.2). The mass squareds of the six mass eigenstates are

$$
\begin{aligned}
& 0, m_{Q}^{2}, m_{Q}^{2}+x_{Q}^{2}|H|^{2}, m_{Q}^{2}+x_{Q}^{2}\left|H^{\prime}\right|^{2}, \\
& \frac{1}{2}\left(m_{Q}^{2}+x_{Q}^{2}|H|^{2}+x_{Q}^{2}\left|H^{\prime}\right|^{2} \pm \sqrt{\left(m_{Q}^{2}+x_{Q}^{2}|H|^{2}+x_{Q}^{2}\left|H^{\prime}\right|^{2}\right)^{2}+4 x_{Q}^{4}|H|^{2}\left|H^{\prime}\right|^{2}}\right) .
\end{aligned}
$$

With a similar computation to the gauge contribution, we find that

$$
\begin{gathered}
V(H) /|H|^{4} \simeq-\frac{3}{32 \pi^{2}} y_{t}^{4}\left(1+4 \ln \frac{|H|}{v^{\prime}}+4 f_{54}\left(\frac{x_{Q}}{y_{t}}\right)\right), \\
f_{54}(r)=r^{4}-\ln r^{2}+\left(r^{6}-\frac{r^{4}}{2}\right) \ln \left(1-\frac{1}{r^{2}}\right) .
\end{gathered}
$$

The one-loop CW potential of the SM from the top quark is

$$
V_{\mathrm{SM}}(H) /|H|^{4}=\lambda_{\mathrm{SM}}(\mu)-\frac{3}{16 \pi^{2}} y_{t}^{4}\left(\ln \frac{y_{t}^{2}|H|^{2}}{\mu^{2}}-\frac{3}{2}\right),
$$

and matching these potentials yields the threshold correction

$$
\lambda_{\mathrm{SM}, \mathrm{t5} 4}\left(v^{\prime}\right) \simeq-\frac{3}{8 \pi^{2}} y_{t}^{4}\left(\ln \frac{e}{y_{t}}+f_{54}\left(\frac{x_{Q}}{y_{t}}\right)\right) .
$$

Note that the threshold correction logarithmically diverges as $x_{Q} \rightarrow y_{t}$ i.e. $m_{Q} \ll x_{Q} v^{\prime}$ because, for $m_{Q} \ll x_{Q} v^{\prime}$, there is an additional particle below the scale $v^{\prime}$ coupling to the SM Higgs.

We next consider the case where the $X$ state for the top quark is $\mathbf{4 5}$, where the relevant interactions are shown in eq. (5.5). We only consider the case $x_{U} \simeq x_{Q}$ and $m_{U} \simeq m_{Q}$, giving the top yukawa coupling

$$
y_{t}=\frac{2 x_{Q}^{2} m_{Q} v^{\prime}}{m_{Q}^{2}+x_{Q}^{2} v^{\prime 2}}
$$

which can be solved for

$$
m_{Q} \simeq \frac{x_{Q}^{2} v^{\prime}}{y_{t}}\left(1 \pm \sqrt{1-\frac{y_{t}^{2}}{x_{Q}^{2}}}\right) .
$$

We find the threshold correction

$$
\lambda_{\mathrm{SM}, \mathrm{t} 45 \pm}\left(v^{\prime}\right) \simeq-\frac{3}{8 \pi^{2}} y_{t}^{4}\left(\ln \frac{e}{y_{t}}+f_{45 \pm}\left(\frac{x_{Q}}{y_{t}}\right)\right),
$$

where the functions $f_{45 \pm}$ are given by

$$
f_{45 \pm}(r)=\frac{5}{12}+r^{4}+r^{4}\left(-\frac{1}{2}+2 r^{2} \pm 2 r \sqrt{r^{2}-1}\right) \ln \frac{r \pm \sqrt{r^{2}-1}}{2 r}-\frac{1}{2} \ln \left(2 r^{3}\left(r \pm \sqrt{r^{2}-1}\right)\right) .
$$

Here \pm correspond to \pm in eq. (6.16). The function $f_{45}(r)$ nearly vanishes around $r=1$. 


\subsubsection{Threshold correction from other fermions}

The threshold correction from other charged fermions are expected to be negligibly small because the corresponding $m_{X} \gg v^{\prime}$ or $x \ll 1$. An exceptional case arises if the up/charm yukawas, arise from $X_{45}$, and $y_{u, c} \ll 1$ follows from $m_{X} \ll x v^{\prime}$ while $x=O(1)$. We do not consider such a case.

The threshold correction from the neutrino that is in the same $\mathbf{1 6}$ as the top quark can be large since $x \sim 1$ and $m_{X} \sim v^{\prime}$. If the $X$ state for the top quark is $\mathbf{5 4}$, the yukawa coupling of $H$ and $H^{\prime}$ to $\ell$ and $\bar{\ell}$ are of the form (5.12), which is SU(4) symmetric. The threshold correction to $\lambda_{\mathrm{SM}}\left(v^{\prime}\right)$ vanishes at one-loop level. If the $X$ state for the top quark is $\mathbf{4 5}$, the yukawa coupling is of the form

$$
x_{T} H^{\dagger} \ell T_{L}+\frac{1}{2} m_{T} T_{L}^{2}+x_{T} H^{\prime \dagger} \bar{\ell} T_{R}+\frac{1}{2} m_{T} T_{R}^{2}
$$

giving the threshold correction

$$
\begin{aligned}
\lambda_{\mathrm{SM}, \nu 45}\left(v^{\prime}\right)= & \frac{x_{T}^{4}}{128 \pi^{2}} f_{45 \nu}\left(\frac{m_{T}}{x_{T} v^{\prime}}\right) \\
f_{45 \nu}(r)= & -\frac{4\left(6 r^{2}+11\right)}{r^{2}+2}-2\left(4 r^{2}+1\right) \ln 2-2\left(12 r^{2}+5\right) \ln r^{2} \\
& +8\left(2 r^{2}+1\right) \ln \left(r^{2}+1\right)+\frac{r\left(r^{2}+1\right)\left(4 r^{2}+9\right) \ln \frac{r^{2}+\sqrt{r^{2}+2} r+1}{r^{2}-\sqrt{r^{2}+2}+1}}{\left(r^{2}+2\right)^{3 / 2}} .
\end{aligned}
$$

We find this correction to be negligible unless $m_{T} \ll x_{T} v^{\prime}$, which requires $m_{T} \ll m_{Q, U}$ and is disfavored from the bottom-tau ratio.

\subsubsection{Threshold correction from colored Higgses in the 422 theory}

In the 422 theory, colored Higgs have masses around the scale $v^{\prime}$, and contribute to the threshold correction to $\lambda_{\mathrm{SM}}\left(v^{\prime}\right)$. We denote the $(\mathbf{4}, \mathbf{2}, \mathbf{1})$ and $(\overline{\mathbf{4}}, \mathbf{1}, \mathbf{2})$ Higgses, in which the SM Higgs and its partner are embedded, as $\Phi_{\alpha}^{a}$ and $\Phi_{a, \alpha^{\prime}}^{\prime}$, respectively. Here $a, \alpha, \alpha^{\prime}$ are the $\mathrm{SU}(4), \mathrm{SU}(2)_{L}, \mathrm{SU}(2)_{R}$ indices, respectively. The $\mathrm{SU}(4) \times \mathrm{SU}(2)_{L} \times \mathrm{SU}(2)_{R} \times P_{L R}$ invariant potential is given in general by

$$
\begin{aligned}
V= & -m^{2}\left(|\Phi|^{2}+\left|\Phi^{\prime}\right|^{2}\right)+\frac{\lambda}{2}\left(|\Phi|^{4}+\left|\Phi^{\prime}\right|^{4}\right)+y|\Phi|^{2}\left|\Phi^{\prime}\right|^{2} \\
& +\frac{k}{2}\left(\Phi_{\alpha}^{a} \Phi^{b \alpha} \Phi_{a \beta}^{*} \Phi_{b}^{* \beta}+\Phi_{\alpha^{\prime}}^{\prime a} \Phi^{\prime} b \alpha^{\prime} \Phi_{a \beta^{\prime}}^{\prime *} \Phi_{b}^{\prime * \beta^{\prime}}\right) \\
& +\left(\frac{l}{2} \Phi_{\alpha}^{a} \Phi^{b \alpha} \Phi_{a}^{\prime \beta^{\prime}} \Phi_{b \beta^{\prime}}^{\prime}+\text { h.c. }\right)+g \Phi_{\alpha}^{a} \Phi_{b}^{* \alpha} \Phi_{a \beta^{\prime}}^{\prime} \Phi^{\prime * \beta^{\prime}},
\end{aligned}
$$

where $|\Phi|^{2} \equiv \Phi^{a \alpha} \Phi_{a \alpha}^{*}$. The threshold correction is given by [18]

$$
\begin{aligned}
\lambda_{\mathrm{SM}}\left(v^{\prime}\right) & =\frac{1}{64 \pi^{2}}|l|^{2} f_{c}\left(\frac{g}{|l|}, \frac{k}{|l|}\right), \\
f_{c}(x, y) & =\frac{\left(1-(x-y)^{2}\right)^{2}}{6(x-y)^{3}}\left(2(x-y)+(x+y) \ln \frac{y}{x}\right) .
\end{aligned}
$$


The function $f_{c}$ is always negative and is typically $O(1)$. As long as $|\ell|, g, k$ are less than 1 , this contribution is subdominant. If $|\ell|, g, k$ are larger than unity, which leads to strongly coupled Higgses at higher energy scales, this contribution can be large and predicts larger top quark masses. We assume weakly coupled Higgs bosons and neglect the threshold correction from the colored Higgses.

\subsection{Top quark mass, QCD coupling and the Higgs Parity breaking scale}

Let us first clarify the top quark mass we use in this paper. We use the pole top quark mass $m_{t}$, from which we compute the $\overline{\mathrm{MS}}$ top yukawa coupling via [15]

$$
y_{t}\left(m_{t}\right)=0.93690+0.00556\left(\frac{m_{t}}{\mathrm{GeV}}-173.34\right)-0.00042 \frac{\alpha_{s}\left(m_{Z}\right)-0.1184}{0.0007},
$$

where the NNNLO QCD quantum correction is included. The conversion necessarily involves an uncertainty due to the non-perturbative nature of QCD [51-53]. To go beyond the precision limited by this uncertainty, which is expected to be as large as the QCD scale, the top quark mass shown in this paper should be understood as a quantity defined by eq. (6.24). Also, the pole mass measured using hadronic final states suffers from the uncertainty of soft QCD processes including hadronization [54]. We nevertheless show the value suggested in [55], $m_{t}=173.0 \pm 0.4 \mathrm{GeV}$, as a guide.

We compute the running of the SM Higgs quartic coupling following [15]. In figure 6 we show the prediction for the Higgs Parity breaking scale $v^{\prime}$ as a function of the top quark mass for various values of the QCD coupling constant and choices of the $X$ states. In the left panels, we take $x_{Q}=1$. Here $f=0$ is a reference point where the contribution to the threshold correction shown by $f_{45,54}$ is suppressed. For a given top quark mass, the prediction for $v^{\prime}$ is smaller than the one for $f=0$, since $f_{45,54} \lesssim 0$. We find that this is also the case for $X_{45}$ with generic $\left(x_{Q}, x_{U}, m_{U}, m_{Q}\right)$. Thus, for a given $v^{\prime}$, which is fixed by successful unification, we obtain an upper bound on the top quark mass.

We can make a sharper prediction by assuming bottom-tau unification discussed in section 5.6. In the right panels, we take the value of $x_{Q}$ to reproduce the bottom/tau ratio. The predictions for $X_{45}$ are indistinguishable from the one for $f=0$. Here it is assumed that $m_{Q}=m_{U}$. We find that this is still the case for $m_{U} \lesssim m_{Q}$, while for $m_{U}>m_{Q}$ the result approaches that of $X_{54}$. The prediction for $X_{54}$ differs from $f=0$, but not by as much as when $x_{Q}=1$. For this case of the simplest successful $b / \tau$ result, for a given $v^{\prime}$ we have two predictions for the top quark mass, which differ from each other by $0.6-1 \mathrm{GeV}$.

\section{Precise unification and SM parameters}

In section 4 we used gauge coupling unification to predict the unified mass scale $M_{X Y}$ and the Higgs Parity breaking scale $v^{\prime}$ in terms of unified threshold corrections from gauge particles, $r_{X Y}$, and from scalars and fermions, $\Delta$, as shown in figures 3 and 4 . In section 6 , $v^{\prime}$ was predicted by evolution of the SM quartic, including threshold corrections from this Higgs Parity breaking scale that are sensitive to the top quark coupling $x_{Q}$, as shown in figure 6 . By combining these results from sections 4 and 6 , which both depend on whether 

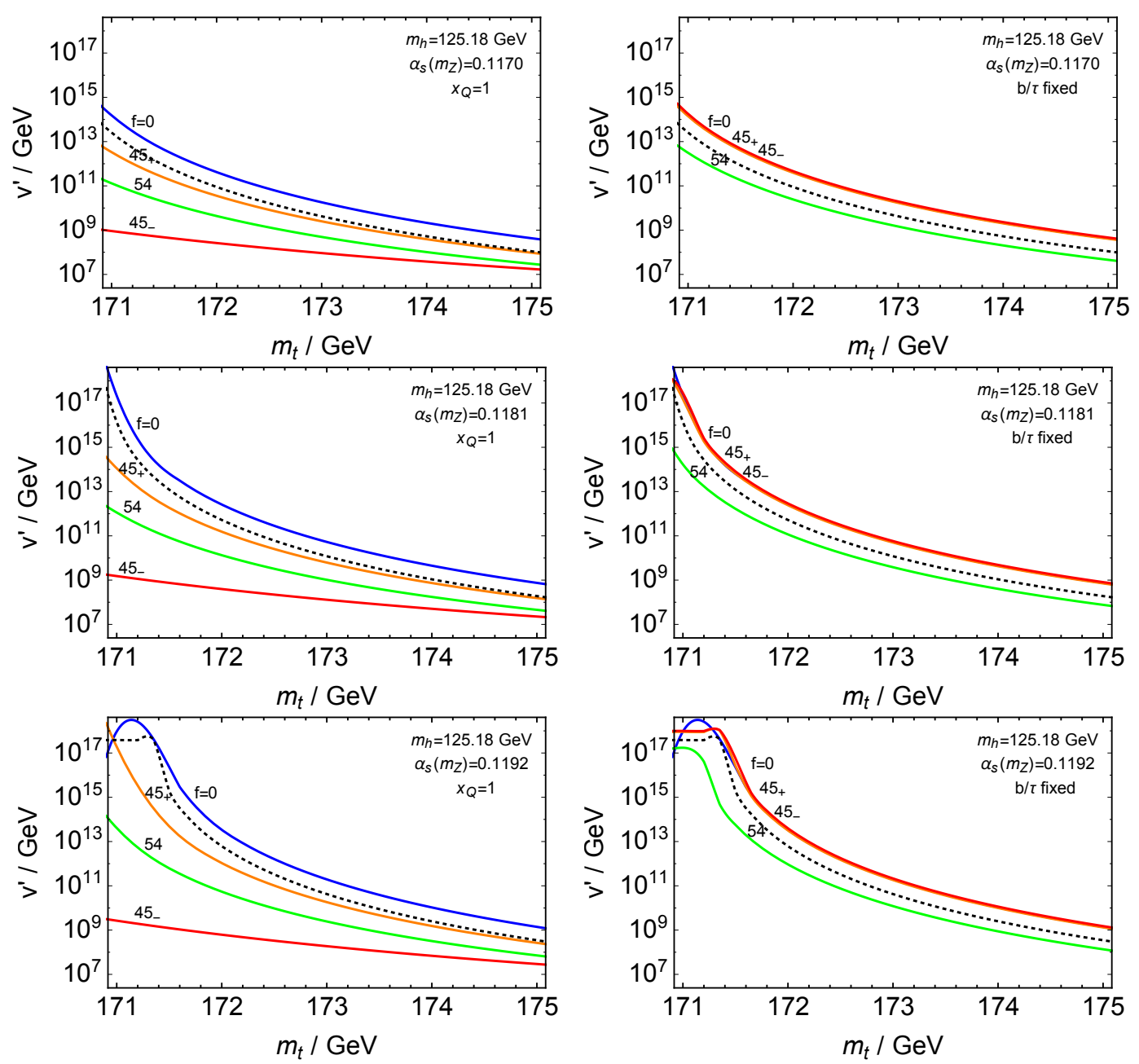

Figure 6. The prediction of $v^{\prime}$, from running of the SM quartic, as a function of $m_{t}$ with the three rows showing different values of $\alpha_{s}$. Dotted lines assume $\lambda_{\mathrm{SM}}\left(v^{\prime}\right)=0$. Solid lines show that the dependence of $v^{\prime}$ on the $X$ states of the top quark is large for $x_{Q}=1$ (left panels), but is reduced when $m_{b} / m_{\tau}$ is imposed (right panels), which also significantly raises the $v^{\prime}$ prediction.

the $X$ state for the top quark mass is a $\mathbf{4 5}$ or $\mathbf{5 4}$, we are finally ready to discuss the correlation among SM parameters discussed in the introduction.

\section{1 $\quad \mathrm{SU}(3) \times \mathrm{SU}(2) \times \mathrm{SU}(2) \times \mathrm{U}(1)$}

In figure 7 , the predicted correlation between $m_{t}$ and $\alpha_{s}\left(m_{Z}\right)$ is shown, for $x_{Q}$ chosen to fix $m_{b} / m_{\tau}$. In the left panel, regions with $\Delta<1$ or 3 are shaded, which is reasonable if the $\mathrm{SO}(10)$ Higgses are $\mathbf{4 5}$ or $\mathbf{5 4}$. For a given theory, $\left(m_{t}, \alpha_{s}\right)$ is predicted with uncertainties of

$$
\begin{aligned}
\delta m_{t} & =0.1 \mathrm{GeV} \times \Delta, \\
\delta \alpha_{s} & \simeq 0.0003 \times \Delta .
\end{aligned}
$$

The $2 \sigma$ range of $m_{t}$ and $\alpha_{s}\left(m_{Z}\right)$ [55] is shown by a dotted box. 

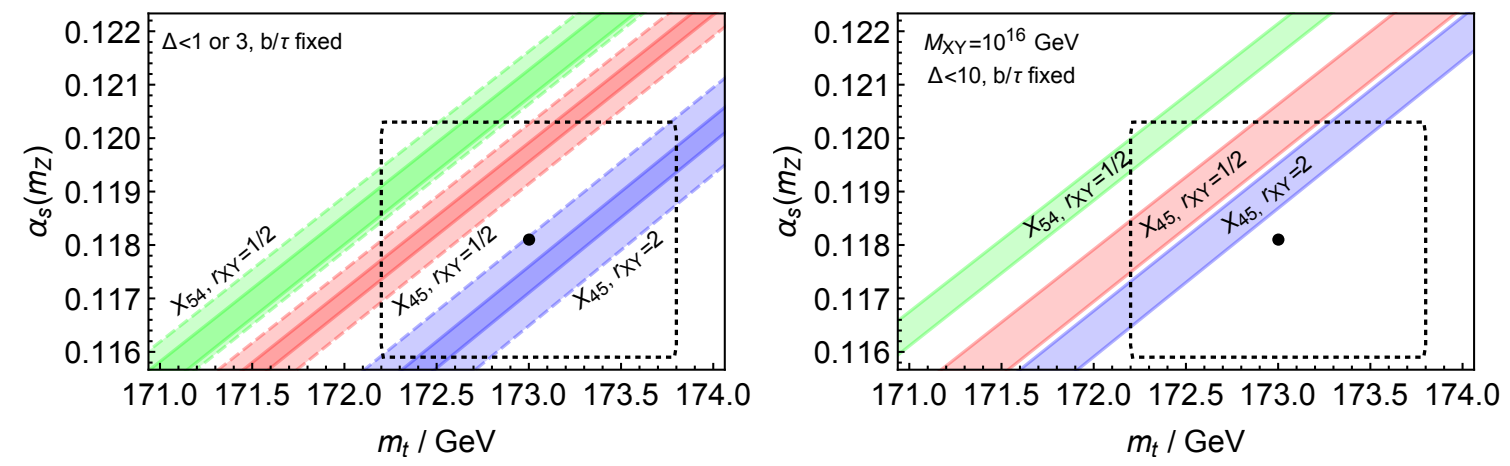

Figure 7. Predicted correlation between the top quark mass $m_{t}$ and the QCD coupling $\alpha_{s}\left(m_{Z}\right)$ in three 3221 models (left panel); with $M_{X Y}$ constrained (right panel). The dot and the dotted box show the central value and the $2 \sigma$ range of the observed values, respectively.

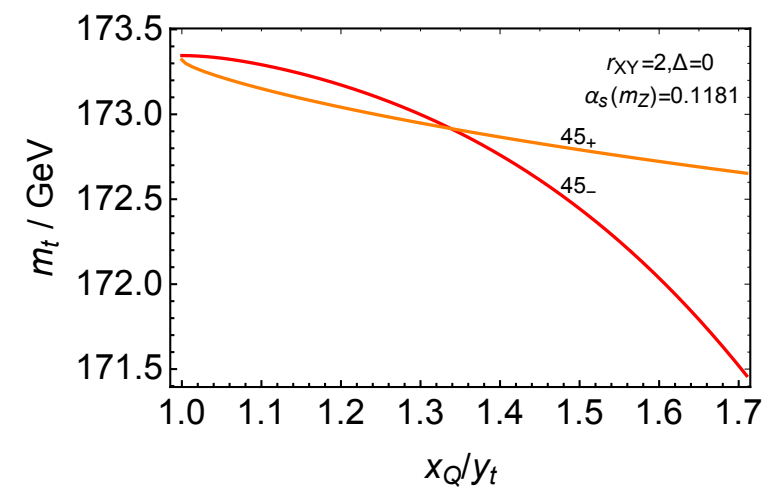

Figure 8. The dependence of the predicted top quark mass $m_{t}$ on $x_{Q}$ in the 3221 theory.

Note that, with $x_{Q}$ fixed by $m_{b} / m_{\tau}$, a top mass from $X_{45}$ gives $f \simeq 0$. Since $f<0$ for $X_{54}$ and $r_{X Y} \leq 2$ for any breaking to 3221 via SO(10) Higgs of $\mathbf{4 5 , 5 4 , 2 1 0}$, the prediction labelled as $X_{45}, r_{X Y}=2$ can be understood as a model-independent upper bound on the top quark mass. For example, if $\alpha_{s}\left(m_{Z}\right)=0.1181$, assuming $\Delta<3$, the top quark mass must be below $173.6 \mathrm{GeV}$. The sensitivity of the prediction on $m_{t}$ to the value of $x_{Q}$ is shown in figure 8 for $X_{45}$ and $r_{X Y}=2$. The prediction on $m_{t}$ decreases by $0.3 \mathrm{GeV}$ if $x_{Q}$ is larger than the one to fix $m_{b} / m_{\tau}$ by more than few $10 \%$.

The running of the gauge and quartic couplings for the experimental central value of $\left(\alpha_{s}, m_{t}\right)$ is shown in figure 1 , assuming $X_{45}, r_{X Y}=2$ and $x_{Q}$ fixing $m_{b} / m_{\tau}$. The global picture of the correlation shown in figure 2 also assumes the same setup, although the picture looks similar for other choices of the $X$ states, $r_{X Y}$ and $x_{Q}$.

In the right panel of figure 7 , we fix the $X Y$ gauge boson mass to be $10^{16} \mathrm{GeV}$, which would be suggested if proton decay is observed by Hyper-K. A large value for $\Delta$ is then needed for unification, and the widths of the shaded bands result from requiring $\Delta<10$. The top quark mass must be below $172.8 \mathrm{GeV}$ for $\alpha_{s}\left(m_{Z}\right)=0.1181$. If proton decay is observed by Hyper-K and the top quark mass is found to be near this bound, we can infer that the bottom-tau ratio is fixed by $x_{Q}$ and $\mathrm{SO}(10)$ symmetry is broken by a $45 \mathrm{VEV}$. 


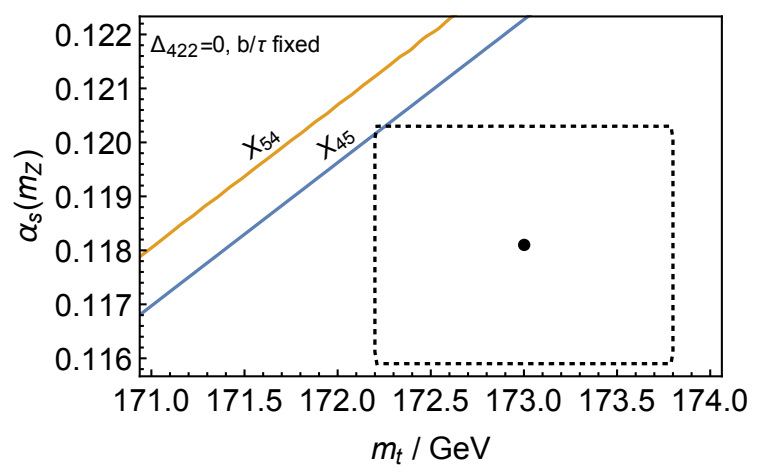

Figure 9. Predicted correlation between the top quark mass $m_{t}$ and the QCD coupling $\alpha_{s}\left(m_{Z}\right)$ in the 422 theory. The dot and the dotted box show the central value and the $2 \sigma$ range of the observed values, respectively.

\section{$7.2 \quad \mathrm{SU}(4) \times \mathrm{SU}(2) \times \mathrm{SU}(2)$}

In the 422 theory, the embedding of the $\mathrm{U}(1)_{Y}$ coupling into the $\mathrm{SU}(4) \times \mathrm{SU}(2)_{R}$ couplings is non-trivial. In the minimal theory, the threshold correction $\Delta_{422}$ only arises from the colored Higgs,

$$
\Delta_{422}=\frac{2}{5} \ln \frac{m_{h_{Q}}}{m_{W^{\prime}}},
$$

where $m_{h_{Q}}$ is the mass of the colored Higgs whose gauge quantum number is the same as that of the SM quark doublet. The magnitude of this correction is less than 1, unless the parameters of the Higgs potential are fine-tuned to make the colored Higgs much lighter than $W^{\prime}$. A contribution to $\Delta_{422}$ may also arise from the mass splitting of $X$ states. As long as $x$ and $m_{X}$ preserve approximate grand unified relations, this contribution is also small. One may wonder whether the hierarchy of $m_{X} \ll x v^{\prime}$ leads to a large threshold correction. This is not the case since the VEV of $H^{\prime}$ breaks $\mathrm{SO}(10)$ only to $\mathrm{SU}(5)$.

For $\Delta_{422}=0, v^{\prime} \simeq 1.3 \times 10^{14} \mathrm{GeV}$ is required. In figure 9 , the predicted correlation between $m_{t}$ and $\alpha_{s}\left(m_{Z}\right)$ is shown, for $x_{Q}$ chosen to fix $m_{b} / m_{\tau}$. Note that, for this choice of $x_{Q}$, a top mass from $X_{45}$ gives $f \simeq 0$. Since $f \lesssim 0$, the prediction labelled as $X_{45}$ can be understood as a model-independent upper bound on the top quark mass. The top quark mass/QCD coupling constant is predicted to be significantly smaller/larger than the central value.

\section{Discussion}

Higgs Parity accounts for a remarkable coincidence: the scale at which the SM quartic coupling vanishes is close to the scale of Left-Right symmetry breaking required for gauge coupling unification in $\mathrm{SO}(10)$, as illustrated in figure 1. In this paper we have explored in detail the precision of this coincidence, which we frame in terms of a correlation of the measured values of the top quark mass and the QCD coupling.

Taking the intermediate gauge symmetry to be 3221, the global picture of this correlation is shown in the right panel of figure 2 , and the fine detail close to the experimental 
values is shown in the left panel of figure 7 . This correlation is indeed remarkable, and appears at least as precise as the correlation of the QCD coupling with the weak mixing angle in supersymmetric unification. The constraint on the 3221 breaking scale from gauge coupling unification alone is shown in figure 3 , and is roughly $v^{\prime} \simeq\left(10^{10}-10^{12}\right) \mathrm{GeV}$. This should be compared with the constraint on $v^{\prime}$ from running the SM quartic coupling, shown in figure 6 , which is significantly affected by the threshold effect from a coupling $x_{Q}$ of the top quark sector. If this parameter is the dominant effect reconciling $m_{b} / m_{\tau}$ with unified yukawa couplings, then this constraint on $v^{\prime}$ is sharpened. Matching the values of $v^{\prime}$ from gauge coupling unification and SM quartic running, and allowing typical threshold corrections in simple models of $\mathrm{SO}(10)$ breaking, then yields a successful prediction at high precision: $\alpha_{s}$ to $1 \%$, or $m_{t}$ to $0.2 \%$, as illustrated in the left panel of figure 7 .

The precision may be reduced in more complicated models, or if large $\mathrm{SO}(10)$ breaking effects enter the spectrum or couplings of the $X$ states that generate yukawa couplings. However, as experimental uncertainties on $\alpha_{s}$ and $m_{t}$ are reduced, evidence may accumulate for a particular simple version of Higgs Parity unification. For example, future measurements leading to the blue region of the left panel of figure 7 would provide evidence for a simple model with: $\mathrm{SO}(10)$ broken via a $\mathbf{4 5}$ to 3221 , small unified corrections from scalars and fermions, $X_{45}$ exchange generating the top yukawa coupling, and $m_{b} / m_{\tau}$ resulting from mixing of states between this $X_{45}$ and the third generation matter $\mathbf{1 6}$.

The dominant sensitivity to $\alpha_{s}$ in this correlation arises from the determination of $v^{\prime}$ from the running of the quartic, not from the determination of $v^{\prime}$ from gauge coupling unification. This implies that the sensitivity of the prediction for $\alpha_{s}$ to the grand unified thresholds, $\Delta_{i}$, as shown by the widths of the shadings in the left panel of figure 7 , is about an order of magnitude less in Higgs Parity unification than in conventional grand unification.

Taking the intermediate gauge symmetry to be 422 leads to a much larger value for $v^{\prime}$ from gauge coupling unification: $v^{\prime} \gtrsim 4 \times 10^{13} \mathrm{GeV}$, even allowing quite large unified threshold corrections, as shown in figure 4. To match the value of $v^{\prime}$ from running of the SM quartic coupling then favors $x_{Q}$ values that successfully determine $m_{b} / m_{\tau}$, but only for large values of $\alpha_{s}$ and small values of $m_{t}$, as shown in figure 9.

In the 3221 theory with minimal content for the $\mathrm{SO}(10)$ breaking Higgs, the unification scale is above $10^{16} \mathrm{GeV}$ and the proton lifetime is predicted to be above the current constraint, as shown in the left panels of figure 3. An observation of proton decay at future experiments would require large threshold corrections at the unification scale, $\Delta \gtrsim 10$, and/or non-minimal $\mathrm{SO}(10)$ breaking. In both cases, a larger $v^{\prime}$ and hence a smaller top quark mass is favored, as illustrated in the right panel of figure 7.

In the 422 theory, threshold corrections at the unification scale from $\mathrm{SO}(10)$ breaking Higgses give $\Delta \sim O(1)$. As figure 4 shows, the theory predicts the unification scale around $10^{15} \mathrm{GeV}$ and hence too short a proton lifetime. The unification scale can be raised to $10^{16} \mathrm{GeV}$ by large threshold corrections, $\Delta>10$, which requires a rich structure around the unification scale such as $\mathrm{SO}(10)$ symmetry breaking induced by supersymmetry breaking.

The observed flavor structure of the SM may arise from an SO(10) unified theory, as suggested in eq. (5.18). Although we have not performed precise fits to the SM fermion 
masses, it would be interesting to do so and to investigate relations between the flavor observables. The model appears to predict a neutrino mass matrix proportional to the up quark mass matrix. However, this is avoided because of mixing between the third generation 16 and $45 / 54$ fermions at the scale $v^{\prime}$. The theory of eq. (5.18) predicts $m_{\nu_{1.2}} \sim\left(v / v^{\prime}\right) m_{u, c}$, while the prediction is smaller by a factor of $\left(1 / 16 \pi^{2}\right)$ if $X_{45}$ is replaced by $X_{54}$; both cases give a normal neutrino mass hierarchy. To obtain realistic neutrino masses requires $v^{\prime}=$ $\left(10^{10}-10^{13}\right) \mathrm{GeV}$, which coincides with the scale required from gauge coupling unification and the vanishing SM quartic coupling. Because of the suppression, the yukawa coupling of the right-handed neutrinos responsible for the see-saw mechanism is larger than naively expected from the see-saw relation, increasing the efficiency of leptogenesis and allowing lower reheat temperatures than usual.

In conventional $\mathrm{SO}(10)$ theories, the amount of fine tuning for symmetry breaking increases as the intermediate scale is reduced below the unification scale. However, with Higgs Parity the amount of fine tuning is independent of the intermediate scale, and corresponds to the usual cost of keeping the weak scale below the cutoff.

\section{Acknowledgments}

This work was supported in part by the Director, Office of Science, Office of High Energy and Nuclear Physics, of the US Department of Energy under Contracts DE-AC02$05 \mathrm{CH} 11231$ (LH) and DE-SC0009988 (KH), as well as by the National Science Foundation under grants PHY-1316783 and PHY-1521446 (LH).

\section{A Contributions of $X$ states to beta functions}

In this appendix we give the contributions of the $X$ states to the beta functions of the gauge couplings at two-loop level. We define the coefficient of the beta function by

$$
\frac{\mathrm{d}}{\mathrm{d} \ln \mu}\left(\begin{array}{c}
\frac{2 \pi}{\alpha_{1}} \\
\frac{2 \pi}{\alpha_{2}} \\
\frac{2 \pi}{\alpha_{3}}
\end{array}\right)=\left(\begin{array}{l}
b_{1} \\
b_{2} \\
b_{3}
\end{array}\right), \quad \frac{\mathrm{d}}{\mathrm{d} \ln \mu}\left(\begin{array}{c}
\frac{2 \pi}{\alpha_{2}} \\
\frac{2 \pi}{\alpha_{4}}
\end{array}\right)=\left(\begin{array}{c}
b_{2} \\
b_{4}
\end{array}\right) .
$$

The contributions of each $X$ multiplet to the coefficients $b_{i}$ of the 3221 theory are

$$
\begin{aligned}
X_{10}: \quad\left(\begin{array}{l}
b_{1} \\
b_{2} \\
b_{3}
\end{array}\right) & =\left(\begin{array}{l}
-\frac{2}{3} \\
-\frac{2}{3} \\
-\frac{2}{3}
\end{array}\right)+\left(\begin{array}{ccc}
-\frac{1}{9} & 0 & -\frac{8}{9} \\
0 & -\frac{29}{6} & 0 \\
-\frac{1}{6} & 0 & -\frac{19}{3}
\end{array}\right)\left(\begin{array}{l}
\frac{\alpha_{1}}{2 \pi} \\
\frac{\alpha_{2}}{2 \pi} \\
\frac{\alpha_{3}}{2 \pi}
\end{array}\right), \\
X_{45}: \quad\left(\begin{array}{l}
b_{1} \\
b_{2} \\
b_{3}
\end{array}\right) & =\left(\begin{array}{l}
-\frac{16}{3} \\
-\frac{16}{3} \\
-\frac{16}{3}
\end{array}\right)+\left(\begin{array}{ccc}
-\frac{10}{3} & -3 & -\frac{32}{3} \\
-1 & -\frac{119}{3} & -8 \\
-\frac{4}{3} & -6 & -\frac{167}{3}
\end{array}\right)\left(\begin{array}{l}
\frac{\alpha_{1}}{2 \pi} \\
\frac{\alpha_{2}}{2 \pi} \\
\frac{\alpha_{3}}{2 \pi}
\end{array}\right), \\
X_{54}: \quad\left(\begin{array}{l}
b_{1} \\
b_{2} \\
b_{3}
\end{array}\right) & =\left(\begin{array}{l}
-8 \\
-8 \\
-8
\end{array}\right)+\left(\begin{array}{ccc}
-\frac{38}{9} & -3 & -\frac{208}{9} \\
-1 & -\frac{131}{3} & -8 \\
-4 & -6 & -91
\end{array}\right)\left(\begin{array}{l}
\frac{\alpha_{1}}{2 \pi} \\
\frac{\alpha_{2}}{2 \pi} \\
\frac{\alpha_{3}}{2 \pi}
\end{array}\right),
\end{aligned}
$$


and for the coefficients $b_{2,4}$ of the 422 theory are

$$
\begin{array}{ll}
X_{10}: \quad\left(\begin{array}{l}
b_{2} \\
b_{4}
\end{array}\right)=\left(\begin{array}{l}
-\frac{2}{3} \\
-\frac{2}{3}
\end{array}\right)+\left(\begin{array}{cc}
-\frac{29}{6} & 0 \\
0 & -\frac{55}{6}
\end{array}\right)\left(\begin{array}{l}
\frac{\alpha_{2}}{2 \pi} \\
\frac{\alpha_{4}}{2 \pi}
\end{array}\right), \\
X_{45}: \quad\left(\begin{array}{l}
b_{2} \\
b_{4}
\end{array}\right)=\left(\begin{array}{l}
-\frac{16}{3} \\
-\frac{16}{3}
\end{array}\right)+\left(\begin{array}{cc}
-\frac{119}{3} & -15 \\
-6 & -\frac{238}{3}
\end{array}\right)\left(\begin{array}{l}
\frac{\alpha_{2}}{2 \pi} \\
\frac{\alpha_{4}}{2 \pi}
\end{array}\right), \\
X_{54}: \quad\left(\begin{array}{l}
b_{2} \\
b_{4}
\end{array}\right)=\left(\begin{array}{l}
-8 \\
-8
\end{array}\right)+\left(\begin{array}{ll}
-73 & -15 \\
-6 & -\frac{221}{2}
\end{array}\right)\left(\begin{array}{l}
\frac{\alpha_{2}}{2 \pi} \\
\frac{\alpha_{4}}{2 \pi}
\end{array}\right) .
\end{array}
$$

\section{B Threshold corrections from $\mathrm{SO}(10)$ breaking scalars}

In this appendix we derive the threshold corrections to the gauge coupling unification from scalar multiplets that spontaneously break $\mathrm{SO}(10)$.

\section{B.1 $\quad \mathrm{SU}(3) \times \mathrm{SU}(2) \times \mathrm{SU}(2) \times \mathrm{U}(1)$}

The smallest representation which can break $\mathrm{SO}(10)$ down to 3221 is $\mathbf{4 5}$. This case is particularly interesting as the strong CP problem is solved by assigning an odd CP parity to $\mathbf{4 5}$. The decomposition of $\mathbf{4 5}$ into non-trivial 3221 representations, and the contribution of each of these to the beta functions, is summarized in table 3 . The representations $(3,2,2,-1 / 3)$ and $(3,1,1,2 / 3)$ are would-be Nambu-Goldstone bosons. The threshold corrections to the gauge couplings are

$$
\begin{aligned}
& \frac{2 \pi}{\alpha_{3}\left(M_{X Y}\right)}=\frac{2 \pi}{\alpha_{10}\left(M_{X Y}\right)}-\frac{1}{2} \ln \frac{m_{(8,1,1)}}{M_{X Y}}+\Delta_{3, G} \\
& \frac{2 \pi}{\alpha_{2}\left(M_{X Y}\right)}=\frac{2 \pi}{\alpha_{10}\left(M_{X Y}\right)}-\frac{1}{3} \ln \frac{m_{(1,3,1)}}{M_{X Y}}+\Delta_{2, G} \\
& \frac{2 \pi}{\alpha_{1}\left(M_{X Y}\right)}=\frac{2 \pi}{\alpha_{10}\left(M_{X Y}\right)}+\Delta_{1, G} .
\end{aligned}
$$

The contributions of $\mathbf{4 5}$ to $\Delta_{i j}=\Delta_{i}-\Delta_{j}$ are

$$
\Delta_{32}=-\frac{1}{2} \ln \frac{m_{(8,1,1)}}{M_{X Y}}+\frac{1}{3} \ln \frac{m_{(1,3,1)}}{M_{X Y}}, \quad \Delta_{31}=-\frac{1}{2} \ln \frac{m_{(8,1,1)}}{M_{X Y}}, \Delta_{21}=-\frac{1}{3} \ln \frac{m_{(1,3,1)}}{M_{X Y}} .
$$

As shown in [56-59], after choosing the parameters of the potential to avoid tachyonic directions, $m_{8,1,1}=m_{1,3,1}=0$. Their masses are given by quantum corrections, taking natural values of about $M_{X Y} / 10$. Even with this hierarchy, $\Delta_{i j}$ are only $\approx 1$.

We also consider $\mathbf{5 4}$ whose decomposition is shown in table 3. Although $\mathbf{5 4}$ can break $\mathrm{SO}(10)$ down only to 422 , its presence allows all components of $\mathbf{4 5}$ to have positive mass squared at tree-level [60]. There are two $(8,1,1)$ representations, from $\mathbf{4 5}$ and $\mathbf{5 4}$, which mix with each other, and we call the mass eigenstates as $(8,1,1)_{1,2}$. The threshold corrections 


\begin{tabular}{|c|c|c|c|c|c|}
\hline $\mathrm{SO}(10)$ & \multicolumn{5}{|c|}{$4 \mathbf{5}$} \\
\hline $\mathrm{SU}(3)$ & 3 & 3 & 8 & 1 & 1 \\
$\mathrm{SU}(2)$ & 2 & 1 & 1 & 3 & 1 \\
$\mathrm{SU}(2)$ & 2 & 1 & 1 & 1 & 3 \\
$\mathrm{U}(1)$ & $-1 / 3$ & $2 / 3$ & 0 & 0 & 0 \\
\hline$-b_{3}$ & $2 / 3$ & $1 / 6$ & $1 / 2$ & 0 \\
$-b_{2}$ & 1 & 0 & 0 & $1 / 3$ \\
$-b_{1}$ & $2 / 3$ & $2 / 3$ & 0 & 0 \\
\hline
\end{tabular}

\begin{tabular}{|c|c|c|c|c|}
\hline $\mathrm{SO}(10)$ & \multicolumn{4}{|c|}{$5 \mathbf{4}$} \\
\hline $\mathrm{SU}(3)$ & 3 & 6 & 8 & 1 \\
$\mathrm{SU}(2)$ & 2 & 1 & 1 & 3 \\
$\mathrm{SU}(2)$ & 2 & 1 & 1 & 3 \\
$\mathrm{U}(1)$ & $-1 / 3$ & $-2 / 3$ & 0 & 0 \\
\hline$-b_{3}$ & $2 / 3$ & $5 / 6$ & $1 / 2$ & 0 \\
$-b_{2}$ & 1 & 0 & 0 & 1 \\
$-b_{1}$ & $2 / 3$ & $4 / 3$ & 0 & 0 \\
\hline
\end{tabular}

\begin{tabular}{|c|c|c|c|c|c|c|c|c|c|c|c|c|}
\hline $\mathrm{SO}(10)$ & \multicolumn{10}{|c|}{$\mathbf{2 1 0}$} \\
\hline $\mathrm{SU}(3)$ & 3 & 3 & 8 & 1 & 1 & 3 & 3 & 8 & 8 & 6 & 3 & 1 \\
$\mathrm{SU}(2)$ & 2 & 1 & 1 & 3 & 1 & 3 & 1 & 3 & 1 & 2 & 2 & 2 \\
$\mathrm{SU}(2)$ & 2 & 1 & 1 & 1 & 3 & 1 & 3 & 1 & 3 & 2 & 2 & 2 \\
$\mathrm{U}(1)$ & $-1 / 3$ & $2 / 3$ & 0 & 0 & 0 & $2 / 3$ & $2 / 3$ & 0 & 0 & $1 / 3$ & $-1 / 3$ & -1 \\
\hline$-b_{3}$ & $2 / 3$ & $1 / 6$ & $1 / 2$ & 0 & & 1 & 3 & $10 / 3$ & $2 / 3$ & 0 \\
$-b_{2}$ & 1 & 0 & 0 & $1 / 3$ & & 2 & $8 / 3$ & 2 & 1 & $1 / 3$ \\
$-b_{1}$ & $2 / 3$ & $2 / 3$ & 0 & 0 & & 4 & 0 & $4 / 3$ & $2 / 3$ & 2 \\
\hline
\end{tabular}

Table 3. Decomposition of 45, $\mathbf{5 4}$ and $\mathbf{2 1 0}$ into representations of 3221. For complex representations, complex conjugations of them are understood.

from $\mathbf{4 5}$ and $\mathbf{5 4}$ are

$$
\left(\begin{array}{c}
\Delta_{32} \\
\Delta_{31} \\
\Delta_{21}
\end{array}\right)=\left(\begin{array}{cccccc}
-\frac{1}{2} & -\frac{1}{2} & \frac{1}{3} & \frac{1}{3} & -\frac{5}{6} & 1 \\
-\frac{1}{2} & -\frac{1}{2} & 0 & 0 & \frac{1}{2} & 0 \\
0 & 0 & -\frac{1}{3} & -\frac{1}{3} & \frac{4}{3} & -1
\end{array}\right)\left(\begin{array}{l}
\ln \frac{m_{(8,1,1)_{1}}}{M_{X Y}} \\
\ln \frac{m_{(8,1,1)_{2}}}{M_{X Y}} \\
\ln \frac{m_{(1,3,1)}}{M_{X Y}} \\
\ln \frac{m_{(3,2,2)}}{M_{X Y}} \\
\ln \frac{m_{(6,1,1)}}{M_{X Y}} \\
\ln \frac{m_{(1,3,3)}}{M_{X Y}}
\end{array}\right)
$$

Here $(3,2,2)$ is in general a linear combination of those from $\mathbf{4 5}$ and $\mathbf{5 4}$ which is physical, while the other linear combination is a would-be Nambu-Goldstone boson.

With $O(1)$ mass splittings, these threshold corrections can be $O(1)$. With mass splittings of $O(10), \Delta$ can be $O(10)$; however such scalar mass hierarchies require fine-tuning of parameters.

We conclude that, in a theory with the strong CP problem solved by Higgs Parity, unified threshold corrections to gauge couplings are typically $O(1)$. However, threshold corrections can be large if the theory is non-minimal or the mass spectrum is fine-tuned, or if significant $\mathrm{SO}(10)$ breaking feeds into the spectrum of $X$ states.

The next smallest representation is $\mathbf{2 1 0}$, whose decomposition is shown in table 3 . This representation breaks $\mathrm{SO}(10)$ down to 3221 without breaking the LR symmetry $C_{L R}$, which is required to maintain Higgs Parity if CP symmetry is not imposed on the theory; see eq. (4.1). One of two $(3,2,2,-1 / 3)$ representations as well as $(3,1,1,2 / 3)$ representation 


\begin{tabular}{|c|c|c|c|c|c|c|c|c|c|}
\hline $\mathrm{SO}(10)$ & \multicolumn{3}{|c|}{$5 \mathbf{S}$} & $\mathrm{SO}(10)$ & \multicolumn{5}{|c|}{$\mathbf{2 1 0}$} \\
\hline $\mathrm{SU}(4)$ & 6 & $20^{\prime}$ & 1 \\
$\mathrm{SU}(2)$ & 2 & 1 & 3 \\
$\mathrm{SU}(2)$ & 2 & 1 & 3 \\
\hline$-b_{4}$ & $2 / 3$ & $4 / 3$ & 0 \\
$-b_{2}$ & 1 & 0 & 1 \\
$\mathrm{SU}(2)$ & 2 & 1 & 3 & 1 & 2 \\
$\mathrm{SU}(2)$ & 2 & 1 & 1 & 3 & 2 \\
\hline$-b_{4}$ & $2 / 3$ & $2 / 3$ & 4 & 4 \\
$-b_{2}$ & 1 & 0 & 5 & $10 / 3$ \\
\hline
\end{tabular}

Table 4. Decomposition of $\mathbf{5 4}$ and $\mathbf{2 1 0}$ into representations of 422 . For complex representations, complex conjugations of them are understood.

are would-be Nambu-Goldstone bosons. The contribution of $\mathbf{2 1 0}$ to $\Delta_{i j}$ is

$$
\left(\begin{array}{c}
\Delta_{32} \\
\Delta_{31} \\
\Delta_{21}
\end{array}\right)=\left(\begin{array}{ccccccc}
-\frac{1}{2} & \frac{1}{3} & 1 & -\frac{1}{3} & -\frac{4}{3} & \frac{1}{3} & \frac{1}{3} \\
-\frac{1}{2} & 0 & 3 & -3 & -2 & 0 & 2 \\
0 & -\frac{1}{3} & 2 & -\frac{8}{3} & -\frac{2}{3} & -\frac{1}{3} & \frac{5}{3}
\end{array}\right)\left(\begin{array}{l}
\ln \frac{m_{(8,1,1)}}{M_{X Y}} \\
\ln \frac{m_{(1,3,1)}}{M_{X Y}} \\
\ln \frac{m_{(3,3,1)}}{M_{X Y}} \\
\ln \frac{m_{(8,3,1)}}{M_{X Y}} \\
\ln \frac{m_{(6,2,2}}{M_{X Y}} \\
\ln \frac{m_{(3,2,2)}}{M_{X Y}} \\
\ln \frac{m_{(1,2,2}}{M_{X Y}}
\end{array}\right) .
$$

Depending on the mass spectrum, $\Delta$ may be as large as 10 even if the mass splittings are of $O(1)$.

\section{B.2 $\mathrm{SU}(4) \times \mathrm{SU}(2) \times \mathrm{SU}(2)$}

The smallest representation which can break $\mathrm{SO}(10)$ down to 422 is $\mathbf{5 4}$. The decomposition of $\mathbf{5 4}$ into the 422 representations and the contribution of each to the beta functions are summarized in table 4 . The threshold corrections to the gauge couplings are

$$
\begin{aligned}
& \frac{2 \pi}{\alpha_{2}\left(M_{X Y}\right)}=\frac{2 \pi}{\alpha_{10}\left(M_{X Y}\right)}-\ln \frac{m_{(1,3,3)}}{M_{X Y}}+\Delta_{2, G} \\
& \frac{2 \pi}{\alpha_{4}\left(M_{X Y}\right)}=\frac{2 \pi}{\alpha_{10}\left(M_{X Y}\right)}-\frac{4}{3} \ln \frac{m_{\left(20^{\prime}, 1,1\right)}}{M_{X Y}}+\Delta_{4, G} .
\end{aligned}
$$

Hence, the contribution of $\mathbf{5 4}$ to $\Delta_{10}$ is

$$
\Delta_{10,54}=\frac{2}{3} \ln \frac{m_{(1,3,3)}}{M_{X Y}}-\frac{4}{3} \ln \frac{m_{\left(20^{\prime}, 1,1\right)}}{M_{X Y}},
$$

which is a few at most, even if the mass splitting is $O(10)$.

The next smallest representation for breaking to 422 is 210, whose decomposition is shown in table 4 . The strong CP problem is solved by assigning an odd CP parity to $\mathbf{2 1 0 .}$ The contribution of $\mathbf{2 1 0}$ to $\Delta_{10}$ is

$$
\Delta_{10,210}=\frac{1}{3} \ln \frac{m_{(15,3,1)}^{3} M_{X Y}}{m_{(15,1,1)}^{2} m_{(10,2,2)}^{2}}=\ln \frac{m_{(15,3,1)}}{M_{X Y}}-\frac{2}{3} \ln \frac{m_{(15,1,1)}}{M_{X Y}}-\frac{2}{3} \ln \frac{m_{(10,2,2)}}{M_{X Y}}
$$

which is at most a few, even if the mass splitting is $O(10)$. 
Open Access. This article is distributed under the terms of the Creative Commons Attribution License (CC-BY 4.0), which permits any use, distribution and reproduction in any medium, provided the original author(s) and source are credited.

\section{References}

[1] ATLAS collaboration, Observation of a new particle in the search for the Standard Model Higgs boson with the ATLAS detector at the LHC, Phys. Lett. B 716 (2012) 1 [arXiv: 1207.7214] [INSPIRE].

[2] CMS collaboration, Observation of a New Boson at a Mass of $125 \mathrm{GeV}$ with the CMS Experiment at the LHC, Phys. Lett. B 716 (2012) 30 [arXiv:1207.7235] [INSPIRE].

[3] ATLAS collaboration, Search for squarks and gluinos in final states with jets and missing transverse momentum using $36 \mathrm{fb}^{-1}$ of $\sqrt{s}=13 \mathrm{TeV}$ pp collision data with the ATLAS detector, Phys. Rev. D 97 (2018) 112001 [arXiv:1712.02332] [INSPIRE].

[4] CMS collaboration, Search for natural and split supersymmetry in proton-proton collisions at $\sqrt{s}=13 \mathrm{TeV}$ in final states with jets and missing transverse momentum, JHEP 05 (2018) 025 [arXiv: 1802.02110] [INSPIRE].

[5] H. Georgi, H.R. Quinn and S. Weinberg, Hierarchy of Interactions in Unified Gauge Theories, Phys. Rev. Lett. 33 (1974) 451 [inSPIRE].

[6] H. Georgi and S.L. Glashow, Unity of All Elementary Particle Forces, Phys. Rev. Lett. 32 (1974) 438 [INSPIRE].

[7] M.S. Chanowitz, J.R. Ellis and M.K. Gaillard, The Price of Natural Flavor Conservation in Neutral Weak Interactions, Nucl. Phys. B 128 (1977) 506 [inSPIRE].

[8] ALEPH collaboration, Measurement of electroweak parameters from $Z$ decays into Fermion pairs, Z. Phys. C 48 (1990) 365 [InSPIRE].

[9] S. Dimopoulos, S. Raby and F. Wilczek, Supersymmetry and the Scale of Unification, Phys. Rev. D 24 (1981) 1681 [INSPIRE].

[10] S. Dimopoulos and H. Georgi, Softly Broken Supersymmetry and SU(5), Nucl. Phys. B 193 (1981) 150 [INSPIRE].

[11] N. Sakai, Naturalness in Supersymmetric Guts, Z. Phys. C 11 (1981) 153 [InSPIRE].

[12] L.E. Ibáñez and G.G. Ross, Low-Energy Predictions in Supersymmetric Grand Unified Theories, Phys. Lett. B 105 (1981) 439 [InSPIRE].

[13] M.B. Einhorn and D.R.T. Jones, The Weak Mixing Angle and Unification Mass in Supersymmetric SU(5), Nucl. Phys. B 196 (1982) 475 [INSPIRE].

[14] W.J. Marciano and G. Senjanović, Predictions of Supersymmetric Grand Unified Theories, Phys. Rev. D 25 (1982) 3092 [InSPIRE].

[15] D. Buttazzo, G. Degrassi, P.P. Giardino, G.F. Giudice, F. Sala, A. Salvio et al., Investigating the near-criticality of the Higgs boson, JHEP 12 (2013) 089 [arXiv:1307.3536] [INSPIRE].

[16] L.J. Hall and Y. Nomura, Grand Unification and Intermediate Scale Supersymmetry, JHEP 02 (2014) 129 [arXiv:1312.6695] [INSPIRE].

[17] L.J. Hall, Y. Nomura and S. Shirai, Grand Unification, Axion and Inflation in Intermediate Scale Supersymmetry, JHEP 06 (2014) 137 [arXiv:1403.8138] [INSPIRE]. 
[18] L.J. Hall and K. Harigaya, Implications of Higgs Discovery for the Strong CP Problem and Unification, JHEP 10 (2018) 130 [arXiv:1803.08119] [INSPIRE].

[19] D. Dunsky, L.J. Hall and K. Harigaya, Higgs Parity, Strong CP and Dark Matter, JHEP 07 (2019) 016 [arXiv: 1902.07726] [INSPIRE].

[20] H. Georgi, The State of the Art-Gauge Theories, Particles and fields - 1974, Proceedings of the Williamsburg Meeting of APS/DPF, Williamsburg U.S.A. (1974), AIP Conf. Proc. 23 (1975) 575 .

[21] H. Fritzsch and P. Minkowski, Unified Interactions of Leptons and Hadrons, Annals Phys. 93 (1975) 193 [INSPIRE].

[22] J.C. Pati and A. Salam, Lepton Number as the Fourth Color, Phys. Rev. D 10 (1974) 275 [Erratum ibid. D 11 (1975) 703] [INSPIRE].

[23] M.A.B. Beg and H.S. Tsao, Strong P, T Noninvariances in a Superweak Theory, Phys. Rev. Lett. 41 (1978) 278 [INSPIRE].

[24] R.N. Mohapatra and G. Senjanović, Natural Suppression of Strong p and t Noninvariance, Phys. Lett. B 79 (1978) 283 [INSPIRE].

[25] T.W.B. Kibble, G. Lazarides and Q. Shafi, Strings in SO(10), Phys. Lett. B 113 (1982) 237 [INSPIRE].

[26] D. Chang, R.N. Mohapatra and M.K. Parida, Decoupling Parity and SU(2)-R Breaking Scales: A New Approach to Left-Right Symmetric Models, Phys. Rev. Lett. 52 (1984) 1072 [INSPIRE].

[27] D. Chang, R.N. Mohapatra and M.K. Parida, A New Approach to Left-Right Symmetry Breaking in Unified Gauge Theories, Phys. Rev. D 30 (1984) 1052 [InSPIRE].

[28] G. Lazarides and Q. Shafi, Superconducting membranes, Phys. Lett. B 159 (1985) 261 [INSPIRE].

[29] S.M. Barr, D. Chang and G. Senjanović, Strong CP problem and parity, Phys. Rev. Lett. 67 (1991) 2765 [INSPIRE].

[30] R. Kuchimanchi, Solution to the strong CP problem: Supersymmetry with parity, Phys. Rev. Lett. 76 (1996) 3486 [hep-ph/9511376] [INSPIRE].

[31] R.N. Mohapatra and A. Rasin, Simple supersymmetric solution to the strong CP problem, Phys. Rev. Lett. 76 (1996) 3490 [hep-ph/9511391] [INSPIRE].

[32] R.N. Mohapatra and A. Rasin, A Supersymmetric solution to CP problems, Phys. Rev. D 54 (1996) 5835 [hep-ph/9604445] [INSPIRE].

[33] R.N. Mohapatra, A. Rasin and G. Senjanović, $P, C$ and strong $C P$ in left-right supersymmetric models, Phys. Rev. Lett. 79 (1997) 4744 [hep-ph/9707281] [INSPIRE].

[34] R. Kuchimanchi, P/CP Conserving CP/P Violation Solves Strong CP Problem, Phys. Rev. D 82 (2010) 116008 [arXiv: 1009.5961] [INSPIRE].

[35] R.T. D'Agnolo and A. Hook, Finding the Strong CP problem at the LHC, Phys. Lett. B 762 (2016) 421 [arXiv:1507.00336] [INSPIRE].

[36] A. Albaid, M. Dine and P. Draper, Strong CP and SUZ , JHEP 12 (2015) 046 [arXiv: 1510.03392] [INSPIRE]. 
[37] K.S. Babu, B. Dutta and R.N. Mohapatra, A theory of $R\left(D^{*}, D\right)$ anomaly with right-handed currents, JHEP 01 (2019) 168 [arXiv:1811.04496] [INSPIRE].

[38] Y. Mimura, R.N. Mohapatra and M. Severson, Grand unified parity solution to the strong CP problem, Phys. Rev. D 99 (2019) 115025 [arXiv:1903.07506] [INSPIRE].

[39] K.S. Babu and R.N. Mohapatra, CP Violation in Seesaw Models of Quark Masses, Phys. Rev. Lett. 62 (1989) 1079 [INSPIRE].

[40] K.S. Babu and R.N. Mohapatra, A Solution to the Strong CP Problem Without an Axion, Phys. Rev. D 41 (1990) 1286 [INSPIRE].

[41] V. Agrawal, S.M. Barr, J.F. Donoghue and D. Seckel, Viable range of the mass scale of the standard model, Phys. Rev. D 57 (1998) 5480 [hep-ph/9707380] [INSPIRE].

[42] L.J. Hall, D. Pinner and J.T. Ruderman, The Weak Scale from BBN, JHEP 12 (2014) 134 [arXiv: 1409.0551] [INSPIRE].

[43] D.R.T. Jones, The Two Loop $\beta$-function for a $G_{1} \times G_{2}$ Gauge Theory, Phys. Rev. D 25 (1982) 581 [INSPIRE].

[44] Super-Kamiokande collaboration, Search for proton decay via $p \rightarrow e^{+} \pi^{0}$ and $p \rightarrow \mu^{+} \pi^{0}$ in 0.31 megaton - years exposure of the Super-Kamiokande water Cherenkov detector, Phys. Rev. D 95 (2017) 012004 [arXiv:1610.03597] [INSPIRE].

[45] Hyper-Kamiokande collaboration, Hyper-Kamiokande Design Report, arXiv: 1805.04163 [INSPIRE].

[46] M. Fukugita and T. Yanagida, Baryogenesis Without Grand Unification, Phys. Lett. B 174 (1986) 45 [INSPIRE].

[47] G.F. Giudice, A. Notari, M. Raidal, A. Riotto and A. Strumia, Towards a complete theory of thermal leptogenesis in the SM and MSSM, Nucl. Phys. B 685 (2004) 89 [hep-ph/0310123] [INSPIRE].

[48] W. Buchmüller, P. Di Bari and M. Plümacher, Leptogenesis for pedestrians, Annals Phys. 315 (2005) 305 [hep-ph/0401240] [INSPIRE].

[49] M. Ibe, T. Moroi and T. Yanagida, Dark matter and baryon asymmetry of the universe in large-cutoff supergravity, Phys. Lett. B 620 (2005) 9 [hep-ph/0502074] [INSPIRE].

[50] S.R. Coleman and E.J. Weinberg, Radiative Corrections as the Origin of Spontaneous Symmetry Breaking, Phys. Rev. D 7 (1973) 1888 [InSPIRE].

[51] I.I.Y. Bigi, M.A. Shifman, N.G. Uraltsev and A.I. Vainshtein, The Pole mass of the heavy quark. Perturbation theory and beyond, Phys. Rev. D 50 (1994) 2234 [hep-ph/9402360] [INSPIRE].

[52] M. Beneke and V.M. Braun, Heavy quark effective theory beyond perturbation theory: Renormalons, the pole mass and the residual mass term, Nucl. Phys. B 426 (1994) 301 [hep-ph/9402364] [INSPIRE].

[53] M. Beneke, Renormalons, Phys. Rept. 317 (1999) 1 [hep-ph/9807443] [INSPIRE].

[54] P.Z. Skands and D. Wicke, Non-perturbative QCD effects and the top mass at the Tevatron, Eur. Phys. J. C 52 (2007) 133 [hep-ph/0703081] [INSPIRE].

[55] Particle Data Group collaboration, Review of Particle Physics, Phys. Rev. D 98 (2018) 030001 [INSPIRE]. 
[56] M. Yasue, Symmetry Breaking of $\mathrm{SO}(10)$ and Constraints on Higgs Potential. 1. Adjoint (45) and Spinorial (16), Phys. Rev. D 24 (1981) 1005 [inSPIRE].

[57] M. Yasue, How to break $\mathrm{SO}(10)$ via $\mathrm{SO}(4) \times \mathrm{SO}(6)$ down to $\mathrm{SU}(2)_{L} \times \mathrm{SU}(3)_{C} \times \mathrm{U}(1)$, Phys. Lett. B 103 (1981) 33 [INSPIRE].

[58] G. Anastaze, J.P. Derendinger and F. Buccella, Intermediate symmetries in the $\mathrm{SO}(10)$ model with $(16+16)+45$ Higgses, Z. Phys. C 20 (1983) 269 [InSPIRE].

[59] S. Bertolini, L. Di Luzio and M. Malinsky, On the vacuum of the minimal nonsupersymmetric SO(10) unification, Phys. Rev. D 81 (2010) 035015 [arXiv:0912.1796] [InSPIRE].

[60] K.S. Babu and E. Ma, Symmetry Breaking in $\mathrm{SO}(10)$ : Higgs Boson Structure, Phys. Rev. D 31 (1985) 2316 [INSPIRE]. 